\title{
Spectroscopic characteristics of polar plumes
}

\author{
G. Del Zanna ${ }^{1, \star}$, B. J. I. Bromage ${ }^{2}$, and H. E. Mason ${ }^{1}$ \\ 1 Department of Applied Mathematics and Theoretical Physics, University of Cambridge, UK \\ 2 Centre for Astrophysics, University of Central Lancashire, Preston, UK
}

Received 14 August 2002 / Accepted 5 November 2002

\begin{abstract}
Extreme ultraviolet observations of plumes in polar coronal holes are presented and their spectroscopic signatures discussed. The study focuses on the base of plumes seen on the disk of the Sun with the Coronal Diagnostic Spectrometer (CDS) on the Solar and Heliospheric Observatory (SOHO) satellite. Spectroscopic diagnostic techniques are applied to characterise the plumes in terms of density, temperature, emission measure and element abundance. Attention is drawn to the particular limitations of some of the techniques when applied to plume structures. In particular, we revisit the Widing \& Feldman (1992) findings of a plume having a large first ionization potential (FIP) effect of 10, showing that instead the Skylab data are consistent with no FIP effect. We present for the first time CDS-GIS (grazing incidence spectrometer) observations of a plume. These observations have been used to confirm the results obtained from normal incidence (NIS) observations. We find that polar plumes exhibit the same characteristics as the Elephant's Trunk equatorial plume. The most striking characteristic of the plume bases is that they are near-isothermal with a peak emission measure at transition region temperatures $\simeq 8 \times 10^{5} \mathrm{~K}$. At these temperatures, plumes have averaged densities $N_{\mathrm{e}} \simeq 1.2 \times 10^{9} \mathrm{~cm}^{-3}$, about twice the value of the surrounding coronal hole region. Element abundances in the plumes are found to be close to photospheric, with the exception of neon which appears to be depleted by 0.2 dex relative to oxygen. The absence of a significant FIP effect in plumes is consistent with fast solar wind plasma, although it is not sufficient to prove a link between the two. Finally, we present a comparison between GIS spectra and the SOHO EIT (EUV Imaging Telescope) broad-band images, showing that temperatures derived from the EIT ratio technique are largely overestimated, for plumes and coronal holes. This is partly due to the fact that the so called "Fe XII $195 \AA$ " and "Fe XV $284 \AA$ А" filters are not isothermal, and in coronal holes and plumes lower-temperature lines dominate the EIT signal.
\end{abstract}

Key words. Sun: corona - techniques: spectroscopic - Sun: transition region - Sun: abundances

\section{Introduction}

Plumes are an intrinsic feature of polar coronal holes. They appear in white-light coronagraph and eclipse images as raylike structures, extending up to many solar radii above the limb of the Sun (see e.g. Van de Hulst 1950; Saito 1965). From their morphology they appear to be tracing the open field lines emerging from the coronal holes. The characteristic cometlike shape at the base of the plumes is best seen in the polar holes where the geometry is most favourable. Here, they can be observed in extreme ultraviolet (EUV) images and easily identified from their shape and enhanced intensity relative to the coronal hole (e.g. Ahmad \& Withbroe 1977; Widing \& Feldman 1992; Walker et al. 1993). They are also seen at X-ray wavelengths (Ahmad \& Webb 1978). By tracing plume structures from the lower corona up to $15 R_{\odot}$, DeForest et al. (1997, 2001) have argued that the plumes seen in white-light and in the EUV are different parts of the same structure. In this paper, we only discuss the base of the plumes as observed in EUV,

Send offprint requests to: G. Del Zanna,

e-mail: G.Del-Zanna@damtp.cam.ac.uk

* Previous address: Centre for Astrophysics, University of Central Lancashire, Preston, UK. projected against the solar disk or very close $\left(R<1.1 \times R_{\odot}\right)$ to the limb.

Although more difficult to identify at low latitude where the plume may be viewed more or less "end-on", masking the plume-like shape, there have been some tentative reports of possible identifications in low-latitude holes viewed near the limb (Wang \& Sheeley 1995; Woo 1996). Del Zanna \& Bromage (1999b) reported the first detection by SOHO of a low-latitude plume visible on the disk, seen in the Elephant's Trunk equatorial coronal hole of 1996. This plume was identified by its spectroscopic characteristics, rather than its morphology. Here, we present observations of polar plumes, showing that they exhibit similar characteristics to the low-latitude plume seen in the Elephant's Trunk.

Before the advent of SOHO, it was not possible to distinguish clearly between the plasma characteristics of plumes and those of the surrounding coronal hole (inter-plume) regions because of the limitations of the observations. Density and temperature measurements in plumes were mostly based on coronagraph or Skylab data from just above the limb (e.g. Ahmad \& Webb 1978). Using white light coronagraph observations and applying the Van de Hulst (1950) technique, the plume densities were estimated to be 3-5 times higher than the surrounding coronal hole. Widing \& Feldman (1992) used a Mg VIII 
density diagnostic ratio on Skylab data but were not able to obtain consistent density values for plumes, due to insufficient spectral resolution. Temperatures were estimated from intensity gradients of EUV emission lines, assuming hydrostatic equilibrium and ionization equilibrium (Widing \& Feldman 1992). Ahmad \& Webb (1978) derived a mean temperature of $8 \times 10^{5} \mathrm{~K}$ (at $1.07 R_{\odot}$ ) for some polar plumes observed by the Skylab S-054 experiment. At this time there was no concensus on the subject of the temperature of plumes; it was not clear whether they were cooler or hotter than the inter-plume regions. The confusion may be due in part to the fact that plumes are sometimes seen to have a very bright base, where the density and temperature are enhanced relative to those in the ambient coronal hole and the main body of the plume (Bromage et al. 1997, 2000; Young et al. 1999). In other cases (including the examples presented here), the plumes appear more diffuse overall, with a near-isothermal temperature which is lower than that in the surrounding coronal hole (e.g. Del Zanna \& Bromage 1999b). This variation in the nature of the plumes probably represents two different stages in the evolution of the structure, with a magnetic reconnection event occurring initially at the base, increasing the density and temperature there. Subsequently a more diffuse structure develops which may fade over a timescale of about a day. Lamy et al. 1997) have shown a tendency for plumes to recur at the same location after disappearing for a time.

It is now firmly established that coronal holes are the source of the fast solar wind, as already pointed out by Noci (1973) and Krieger et al. (1973). The question now is how it is generated and whether plumes might be the source.

The SOHO spectroscopic instruments (CDS, SUMER and UVCS) have provided improved spatial and spectral resolution, allowing more detailed analyses to be made in the plume/interplume regions. Hassler et al. (1999) reported finding blue-shifts in SUMER (Solar Ultraviolet Measurements of Emitted Radiation) spectra from the coronal hole network junctions, while Giordano et al. (2000) and Patsourakos \& Vial (2000) have found evidence from UVCS (Ultraviolet Coronagraph Spectrometer) and SUMER observations, respectively, that the fast solar wind is accelerated in the interplume regions. Similar results were recently found by Teriaca et al. (2002), also using UVCS and SUMER observations.

Solar wind models depend heavily on the density and temperature of the source region. The uncertainty in these quantities obtained from the early observations of plumes is reflected in the variety of different theoretical solar wind-plume models which have been proposed (e.g. Wang 1994; Velli et al. 1994; Del Zanna et al. 1997; Casalbuoni et al. 1999). More direct measurements of electron densities have now become possible, using spectral line ratios from SOHO data. Densities derived in this way are more accurate, although it should be kept in mind that these are averaged values (for the emitting plasma along the line of sight).

Wilhelm et al. (1998) presented off-limb (in the range 1.03-1.6 $R_{\odot}$ ) SUMER spectroscopic observations of plume and interplume regions. Densities of both plume and interplume regions were estimated using Si VIII line ratios, and found to start at $N_{\mathrm{e}} \simeq 1 \times 10^{8} \mathrm{~cm}^{-3}$ and then decrease with height, with plumes having shallower density gradients. A limitation of this study was that the plume and interplume observations were not simultaneous, but were separated in time by about six months. They also determined plume and interplume temperatures, using a Mg IX line ratio, finding temperature profiles in the plumes which initially appeared constant at around $T=7.8 \times 10^{5} \mathrm{~K}$ but decreased further out, while in interplume regions the temperature tended to increase steadily with height. SUMER and UVCS off-limb observations (cf. Hassler et al. 1997; Noci et al. 1997) have shown broader spectral line widths in the interplume lanes than in the plumes, indicating lower temperatures in the plumes. Such off-limb spectral observations may suffer from contamination by foreground or background emission, in particular, plume emission behind the coronal hole (interplume) regions can affect measurements of densities and temperatures. Similarly, in the "plume" regions observed off-limb, there may be contamination from "interplume" plasma, or from other plumes present along the line of sight.

DeForest et al. (1997) used EIT images to estimate plume temperatures. They found them to be in the range $1-1.5 \times 10^{6} \mathrm{~K}$, while the interplume temperatures appeared higher (by no more than $30 \%$ ). Problems with this technique are discussed in detail below (Sect. 4). Del Zanna \& Bromage (1999b) found the Elephant's Trunk plume to be cooler than the surrounding coronal hole, with a temperature of about $7.8 \times 10^{5} \mathrm{~K}$.

Another parameter which can be determined spectroscopically is the composition of the coronal plasma. This can provide a link between the fast solar wind and regions which might be its source. Coronal abundances have been found to differ from photospheric values in a way which appears to be related to the first ionization potential (FIP) of the various elements (see the reviews of Feldman 1992, and Raymond et al. 2001). The degree of this "FIP effect" can vary depending on the coronal structure being observed. Early observations revealed that the slow solar wind had a FIP effect of about 4, with low-FIP $(\leq 10 \mathrm{eV})$ elements more abundant than the high-FIP ones. On the other hand, the fast solar wind exhibited abundances closer to photospheric values. A recent study (Von Steiger et al. 2000), based on a re-analysis of the Ulysses data has confirmed that the fast solar wind has photospheric abundances, but suggests an average FIP effect of about 2 for the slow solar wind.

The only pre-SOHO measurement of plume abundances is that of Widing \& Feldman (1992), who studied the brightest polar plume observed by the Skylab S082A EUV spectroheliograph, in December 1973. They derived one of the largest FIP biases reported (a factor of 10). Since then, it has long been thought that plumes have a large FIP effect. If this is the case, then plumes cannot be the major contributors to the fast solar wind. However, a new analysis of the Skylab data is presented here (Sect. 2.2), which shows the FIP effect to be very small or even absent in the plume.

More recent studies using SOHO-CDS data indicate that plumes exhibit only a small FIP effect (Del Zanna \& Bromage 1999a; Del Zanna 1999; Del Zanna \& Bromage 1999b; Young et al. 1999). However, problems with the instrument calibration led to uncertainties of about a factor of two in the derived abundances. The CDS calibration has now been substantially 
improved (see Del Zanna et al. 2001a and Del Zanna 2002) thus allowing a more accurate determination of the FIP effect in plumes.

We have observed a large number of polar plumes with CDS, during solar minimum conditions in 1996, and in 1997. In this paper, we present CDS observations of two "typical" polar plumes seen on the disk. These were chosen in order to focus attention on the base of plumes, and to avoid problems of contamination associated with off-limb measurements. The principal aims of this paper are:

(a) to identify the characteristic signature of a plume in CDS spectra;

(b) to demonstrate that plumes can also be characterised spectroscopically in terms of their density, temperature and composition;

(c) to show that polar plumes, identified from EIT images, exhibit the same spectral characteristics as the low-latitude plume seen in the Elephant's Trunk coronal hole of 1996;

(d) to examine the spectroscopic diagnostic techniques applied to the CDS spectra in order to determine the above quantities, high-lighting the problems encountered, in particular when deducing element abundances (see Sect. 2); and

(e) to explain how it is that polar plumes (which are cool objects) may be seen in the EIT $195 \AA$ and $284 \AA$ images which generally reveal plasma emitting at a temperature of more than $1.5 \times 10^{6} \mathrm{~K}$. In Sect. 4 we present the first in-flight direct study of the emission that contributes to the EIT filters, based on a comparison between EIT and CDS near-simultaneous observations, and we discuss the validity of the EIT filter ratio estimates of temperature.

\section{Measurements of densities, temperatures and element abundances}

For general reviews and definitions on the subject of spectroscopic techniques see Mason \& Monsignori Fossi (1994) and Del Zanna et al. (2002). The intensity $I\left(\lambda_{i j}\right)$, of an optically thin spectral line of wavelength $\lambda_{i j}$ can be written:

$I\left(\lambda_{i j}\right)=\int A_{\mathrm{b}}(X) C\left(T, \lambda_{i j}, N_{\mathrm{e}}\right) \operatorname{DEM}(T) \mathrm{d} T$

where we have defined the differential emission measure $\operatorname{DEM}(T)=N_{\mathrm{e}} N_{\mathrm{H}} \frac{\mathrm{d} h}{\mathrm{~d} T}, A_{b}(X)$ is the element abundance, and $N_{\mathrm{e}}$ and $N_{\mathrm{H}}\left(\mathrm{cm}^{-3}\right)$ are the electron and hydrogen number densities. The contribution function $C\left(T, \lambda_{i j}, N_{\mathrm{e}}\right)$ of each line contains all the atomic parameters and is peaked in temperature, confining the emission to a limited temperature range.

The temperature dependence of the $C(T)$ is mainly provided by the ion fraction term. The conditions in the 'quiet' solar corona are such that ionization equilibrium normally holds, and the $C(T)$ s are calculated using ionization balance calculations. These in turn therefore have a significant role not only in the derivation of the $D E M$, but also in obtaining the element abundances (see below). The ionization equilibrium calculations of Mazzotta et al. (1998) were used here. Note that while the values for the Fe ions are similar to those of Arnaud \& Raymond (1992), for other elements the differences from the earlier calculations of Arnaud \& Rothenflug (1985) are sometime substantial (see Del Zanna 1999, for examples).

The use of different ions from different isoelectronic sequences can help in assessing whether the atomic physics is in disagreement with the observations (e.g. Young et al. 1998). A large number of ions exhibit anomalous behaviour such that lines produced by these ions have theoretical intensities that are consistently under- or over-estimated by large factors. In particular, the anomalous behaviour of the ions of the $\mathrm{Li}$ and $\mathrm{Na}$ isoelectronic sequences has been known for more than 30 years (see the review of Del Zanna et al. 2002), but is still largely neglected. Until the reasons for this anomalous behaviour are understood, lines from ions such as: C IV, Si IV, N V, O VI, Ne VIII, Na IX, Mg X, Al XI, Si XII, S XIV should not be used for any $D E M$ or element abundance analyses, contrary to what is current practice (e.g. Laming \& Feldman 1999).

We have used the CHIANTI atomic database (Dere et al. 1997) to calculate the contribution functions of the observed lines. In particular, Version 3.01 of the database (Dere et al. 2001) was used in this paper for the temperature estimates and for the analysis of the Skylab plume (Sect. 2.2). For the DEM analysis of the plume in Sect. 3.3.1 Version 4.0 of the database (Young et al. 2002) was used. This version, compared to the previous one, includes updates concerning the following ions: O V, Si XI, Mg VI, AL VII, Si VIII. These updates typically only change the emissivities of $10-20 \%$ at maximum, for the lines used here. For the estimates of the electron densities, the new Version 4.0 has been used (see below).

The DEM analysis was performed using a modified version of the Arcetri inversion code (Monsignori Fossi \& Landini 1991). It should be mentioned that the determination of the $D E M$ distribution is an ill-posed problem (see, e.g. Craig \& Brown 1986). However, improvements in the conditioning are made with a careful selection of lines (cf. McIntosh 2000).

\subsection{Elemental abundances}

In principle, from any observed intensity ratio $I_{1} / I_{2}$ it is possible to derive a relative element abundance $A_{\mathrm{b}}\left(X_{1}\right) / A_{\mathrm{b}}\left(X_{2}\right)$ of two elements $X_{1}$ and $X_{2}$ :

$\frac{A_{\mathrm{b}}\left(X_{1}\right)}{A_{\mathrm{b}}\left(X_{2}\right)}=\frac{I_{1} \times \int C_{2}\left(T, N_{\mathrm{e}}\right) \operatorname{DEM}(T) \mathrm{d} T}{I_{2} \times \int C_{1}\left(T, N_{\mathrm{e}}\right) \operatorname{DEM}(T) \mathrm{d} T}$,

if the element abundances are assumed constant along the line of sight and the lines are emitted by the same plasma region. In reality, various assumptions or approximations can account for a variation of a factor of two or more in the derived element abundances (more details can be found in Del Zanna et al. 2001b, and Del Zanna et al. 2002).

The approach adopted in this paper is to derive a $D E M$ curve for each element, and to determine relative element abundances, by normalising the $D E M$ curves of the different elements, as described, for example, in Del Zanna \& Bromage (1999b). On the other hand, when investigating relative abundances, most authors have used (and still use) a "standard approach", which is to use ratios of lines that have contribution functions with similar temperature dependence and which may 
therefore be assumed to come from the same emitting volume. Then a mean value for the DEM over the temperature range of the lines is used in Eq. (2), so that it may be cancelled from the fraction, leaving:

$$
\frac{A_{\mathrm{b}}\left(X_{1}\right)}{A_{\mathrm{b}}\left(X_{2}\right)}=\frac{I_{1} \times \int C_{2}\left(T, N_{\mathrm{e}}\right) \mathrm{d} T}{I_{2} \times \int C_{1}\left(T, N_{\mathrm{e}}\right) \mathrm{d} T} .
$$

Such an approach was first proposed by Widing \& Feldman (1989). Equation (1) is thus approximated by defining for each spectral line a single differential emission measure $<D E M>$ value for the temperature range over which the line forms. For clarity we call this average $<D E M>$ value the line differential emission measure $D E M_{\mathrm{L}}$ :

$D E M_{\mathrm{L}} \equiv\left\langle N_{\mathrm{e}} N_{\mathrm{H}} \frac{\mathrm{d} h}{\mathrm{~d} T}\right\rangle \equiv \frac{I_{\mathrm{ob}}}{A_{\mathrm{b}}(X) \int C(T) \mathrm{d} T}$,

where $I_{\mathrm{ob}}$ is the observed intensity of the line.

Their method (referred to here as the $D E M_{\mathrm{L}}$ method) is to plot the $A_{\mathrm{b}}(X) D E M_{\mathrm{L}}=I_{\mathrm{ob}} / \int C(T) \mathrm{d} T$ values and derive the relative element abundances by adjusting them in order to have all the $D E M_{\mathrm{L}}$ points of the various ions lie along a common smooth curve. The above is actually justified only when:

$\frac{\int C_{1}\left(T, N_{\mathrm{e}}\right) D E M(T) \mathrm{d} T}{\int C_{2}\left(T, N_{\mathrm{e}}\right) D E M(T) \mathrm{d} T}=\frac{\int C_{1}\left(T, N_{\mathrm{e}}\right) \mathrm{d} T}{\int C_{2}\left(T, N_{\mathrm{e}}\right) \mathrm{d} T}$

i.e. if, as well as $C_{1}$ and $C_{2}$ having a similar temperature dependence, $\operatorname{DEM}(T)$ is constant over the temperature range involved.

To study the FIP effect, ratios of lines from low-FIP and high-FIP elements such as $\mathrm{Mg}$ VI/Ne VI and Ca IX/Ne VII are commonly used. In particular, the $\mathrm{Mg} \mathrm{VI} / \mathrm{Ne} \mathrm{VI}$ ratios of the lines around $400 \AA$ (Mg VI: 399.28, 400.67, 403.31 $\AA$; Ne VI $399.82,401.14,403.25,401.93 \AA$ ) have been widely used in solar physics (cf. Sheeley 1996).

There are a number of effects that may result in the abundance ratio not being proportional to the observed intensity ratio or that are potential sources of uncertainties. For example: 1) the $D E M$ effect; 2) the ionization balance or nonequilibrium effects; 3) the use of lines of particular isoelectronic sequences; 4) the density dependence of the lines used.

Considering the first case, if for example the $\operatorname{DEM}(T)$ has a steep gradient in one temperature range, any small differences between $C_{1}$ and $C_{2}$ in that range would be amplified and Eq. (5) would not hold. For example, it is well known that the Mg VI contribution functions are slightly skewed toward higher temperatures, when compared to those of Ne VI (Phillips 1997). The difference is at a maximum just where (log $T=5.9$ ) the $D E M$ gradient is large for plumes. Neglecting the shape of the $D E M$ curves when calculating the $\mathrm{Ne} / \mathrm{Mg}$ relative abundances using $\mathrm{Ne} \mathrm{VI}$ and $\mathrm{Mg}$ VI ions therefore has the effect of underestimating the $\mathrm{Ne} / \mathrm{Mg}$ relative abundance. Del Zanna et al. (2001b) have used the Elephant's Trunk low-latitude plume $D E M$ to show that if Mg VI and Ne VI lines are used, the FIP effect can be substantially overestimated, up to a factor of 3. This DEM effect is even more pronounced when other line ratios such as Ca IX / Ne VII and
Mg VII / Ne VII are considered, because the differences in their $C(T)$ are even larger. Section 2.2 below provides a clear example of this problem. Since observations of different solar regions can have very different $D E M$ distributions, it is impossible to know a priori when the approximation proposed by Widing \& Feldman (1989) is valid.

Finally, a few words about other sources of uncertainties. As already mentioned, the major one is related to the ionization equilibrium calculations. Any inaccuracy could easily amplify the $D E M$ effect. Also, lines of anomalous behaviour should not be used. Nonequilibrium ionization effects can also be non-negligible in some cases, as shown by Edgar \& Esser (2000). Density variations can also affect element abundances if, as in the case of the Mg VI / Ne VI ratios, some of the lines are slightly density-dependent (Mg VI, see Del Zanna et al. 2001b). Transition region densities at temperatures where $\mathrm{Mg}$ VI lines form are difficult to measure, and usually different line ratios produce different results, with average values $N_{\mathrm{e}} \simeq 10^{9} \mathrm{~cm}^{-3}$ (see e.g. Widing \& Feldman 1993; Del Zanna \& Bromage 1999a). Another source of uncertainty is the unknown density vs. temperature variation along the line of sight. Ideally, if enough density estimates at different temperatures are available, one could calculate the emissivities with the appropriate density-temperature model, although other effects can still come into play (see Doschek 1984).

\subsection{The Skylab plume}

We now show the importance of the DEM effect in the derivation of the FIP effect for the plume observed by Widing \& Feldman (1992). A DEM analysis was performed on the calibrated data tabulated in Widing \& Feldman. The line emissivities have been calculated using CHIANTI version 3.01, and the same constant density $\left(1 \times 10^{9} \mathrm{~cm}^{-3}\right)$ adopted by Widing $\&$ Feldman. The main difference compared with their work is the use of the more recent ionization equilibrium calculations of Mazzotta et al. (1998). As a starting point, the photospheric abundances of Grevesse \& Anders (1991) have been used.

Figure 1 shows the $D E M_{\mathrm{L}}$ values for the Skylab plume. Following the $D E M_{\mathrm{L}}$ method, the points are displayed at the temperatures $T_{\max }$, defined as the temperature where $C(T)$ has a maximum. First, we note that the use of $T_{\max }$ can be misleading since, in some cases, this can be quite different from the temperature at which most of the emission occurs. It is often more informative to use the "effective temperature" $T_{\text {eff }}$, defined as $\log T_{\text {eff }}=\int C(T) \operatorname{DEM}(T)$ $\log T \mathrm{~d} T /\left(\int C(T) \operatorname{DEM}(T) \mathrm{d} T\right)$. Figure 1 clearly shows that, in order to align the $D E M_{L}$ points on a smooth curve, it is necessary to modify the adopted $\mathrm{Mg} / \mathrm{Ne}$ relative abundances by a large factor, consistent with the FIP effect found by Widing and Feldman.

A $D E M$ analysis has now been performed on the dataset, and the result displayed as a dashed line in Fig. 1. It is clear that there is a large difference between the $D E M_{\mathrm{L}}$ points and the $D E M$ values. Table A. 1 in the Appendix shows the results in detail. The agreement between theory and observations is good, with the exception of the Ne VII line. It should be kept in 


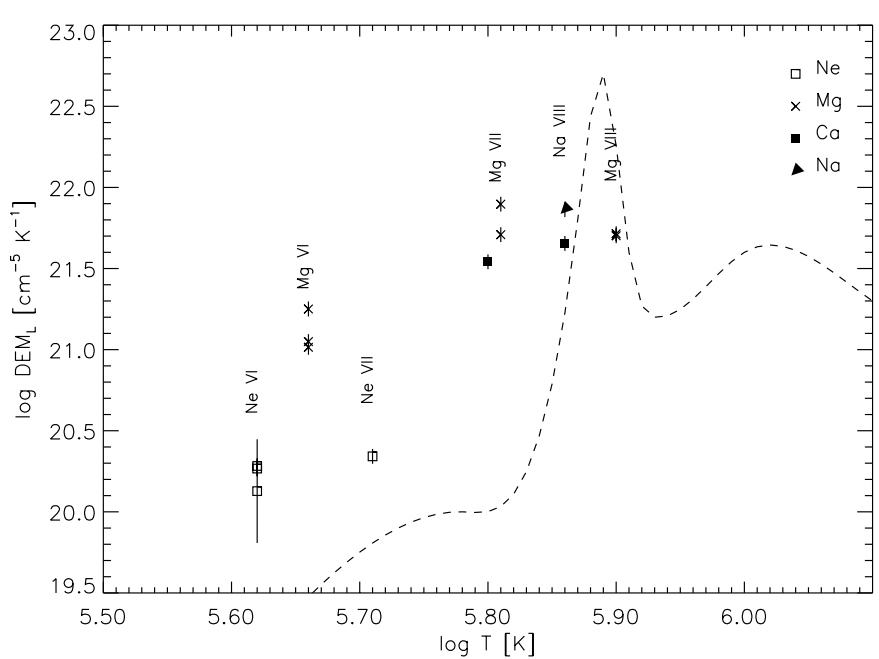

Fig. 1. The $D E M_{\mathrm{L}}$ values for the Skylab plume. The $\mathrm{Ne}$ and $\mathrm{Mg}$ values indicate the need to modify the adopted $\mathrm{Ne} / \mathrm{Mg}$ photospheric abundance ratio, in order to obtain a smooth distribution of $D E M_{\mathrm{L}}$ values. The $\operatorname{DEM}(T)$ derived from the same data is plotted for comparison (dashed line).

mind that the DEM is not constrained at low and high temperatures, due to the lack of observational data. However, the $D E M$ analysis shows that the plume had a near isothermal distribution, similar to that derived by Del Zanna \& Bromage (1999a) for an equatorial plume, with a peak at $\log T=5.9$, and that the use of photospheric abundances results in a good fit to the data, in contrast to the FIP factor of 10 found by Widing \& Feldman. The large difference between our result and that of Widing \& Feldman is mainly due to the fact that the DEM exhibits a steep gradient where the $C(T)$ functions of the Ne VI and $\mathrm{Mg}$ VI lines differ most.

Our results are confirmed by using another emission measure method (see Del Zanna et al. 2002 and references therein for details). This consists of plotting the ratio $I_{\mathrm{ob}} /\left(A_{\mathrm{b}} * C(T)\right)$ for each line as a function of temperature and using the envelope of these curves to constrain the shape of the emission measure distribution and the element abundances. The $I_{\mathrm{ob}} /\left(A_{\mathrm{b}} * C(T)\right)$ curves for the plume, calculated with photospheric abundances, are displayed in Fig. 2. They clearly show that the plasma is nearly isothermal, since all the curves are crossing at one point (with the exception of the Ne VII 465.2 A resonance line, which clearly departs from this behaviour and requires further investigation). Figure 2 also shows that the observed $\mathrm{Mg}$ VI and $\mathrm{Ne} \mathrm{VI}$ intensities are consistent with photospheric abundances.

\subsection{Electron density and temperature}

The CDS wavelength range is rich in density diagnostics (see Mason et al. 1997), but they are mainly confined to hightemperature lines that are not emitted by plumes. The principal exceptions are a few cases. Lower transition region densities have been obtained from O IV lines, while upper transition region - coronal densities have been obtained here from Mg VII, Mg VIII, and Si IX lines, which are good density diagnostics

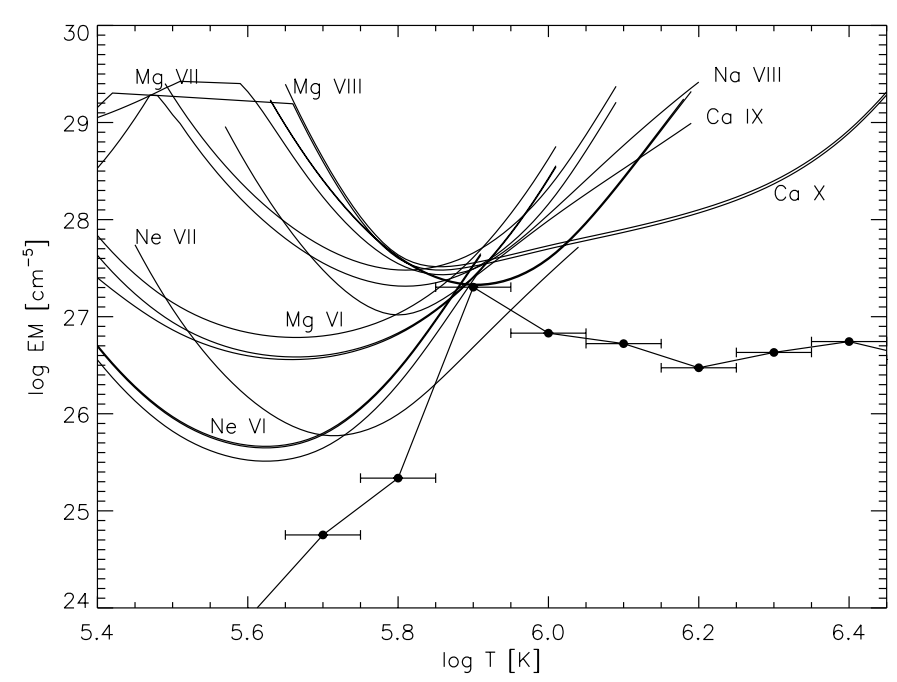

Fig. 2. The $I_{\mathrm{ob}} /\left(A_{\mathrm{b}} * C(T)\right)$ curves for the Skylab plume, assuming photospheric abundances. The data indicate an isothermal distribution at $\log T=5.9$ and are consistent with no FIP effect present. The emission measure $E M(0.1)$ values (see Del Zanna et al. 2002, for the definition), calculated with the $D E M$ of Fig. 1 and a $\Delta \log T=0.1$ are also shown (filled circles).

for coronal holes (see e.g. Del Zanna \& Bromage 1999b and Fludra et al. 1999).

We have used the new Version 4.0 of the CHIANTI database for the calculation of electron densities. This version includes collisional proton excitation in the calculation of the level balance. Proton excitation has a non-negligible effect in the level population calculations of some ions, including Mg VIII, and Si IX.

This in turn affects the emissivities of EUV lines so that to lower the derived densities. Photoexcitation also starts to be non-negligible at some height in the corona, but not at the low heights of the plume bases examined here, so it has been neglected.

The Mg VII, Mg VIII, and Si IX line ratios used here are only marginally sensitive to temperature variations. However, we have calculated the emissivities at the appropriate temperatures derived from line ratios.

Estimates of coronal temperatures (averages over the line of sight) are obtained here from ratios of lines emitted by ions of the same element and close ionization stage, assuming that the plasma is isothermal. The use of different line ratios has been complemented by $D E M$ analyses.

\section{The CDS observations and results}

CDS observations of two polar plumes are presented here. The first was observed on the 23rd August 1996. The second plume was visible during the second week of October 1997. Both plumes were stable structures which lasted for several days in the north polar coronal hole during solar minimum conditions. 


\subsection{The CDS instrument and data analysis}

CDS (Harrison et al. 1995) consists of two instruments: a Normal Incidence Spectrometer (NIS) and a Grazing Incidence Spectrometer (GIS), which cannot both operate at the same time. The NIS detectors observe two spectral ranges simultaneously (NIS 1: 308-379 ̊ and NIS 2: 513-633 $\mathrm{A}$ ), and with the rastering of a long slit can provide monochromatic images of the solar field of view. The GIS produces astigmatic spectra with its four detectors (GIS 1: 151-221 $\mathrm{A}$, GIS 2: 256-341 GIS 3: 393-492 $\AA$ and GIS 4: 659-785 $\AA$ ). GIS spectra are normally recorded using a pinhole slit and it is not usually used for imaging. The CDS observes many emission lines from a large number of highly ionised ions of the most abundant elements, covering a large range of temperatures, and therefore provides a new opportunity to study in detail the transition region and coronal plasma.

The CDS instrument is well suited to the study of element abundances, because the lines observed by CDS are produced by a variety of ions, covering a large temperature range and different isoelectronic sequences. All the low temperature lines $(\log T \leq 5.5 \mathrm{~K})$ observed by $\mathrm{CDS} / \mathrm{NIS}$ are from highFIP elements $(\mathrm{N}, \mathrm{O}, \mathrm{Ne})$ while all the high temperature lines $(\log T \geq 5.9 \mathrm{~K})$ are from low-FIP elements $(\mathrm{Mg}, \mathrm{Ca}, \mathrm{Fe}, \mathrm{Si}$, $\mathrm{Al})$. It is therefore possible to deduce the relative abundances among these two groups of lines. The scaling between the high and low-FIP elements can be achieved using low-FIP, lowtemperature lines and high-FIP, high-temperature lines which overlap in temperature. Unfortunately, NIS observes only a few such lines (one Mg VI line at $349.2 \AA$, which is a complex blend of lines, three Mg VII lines and a few Ne VI and Ne VII lines). It is important to complement the NIS observations with ones made at about the same time with the GIS instrument, as has been done here, since GIS observes many other lines and ions that extend the overlapping region between the high-FIP and the low-FIP ions observed.

A series of standard corrections was applied to the raw NIS data. These include de-biasing, flat-fielding, corrections for the burn-in of a few lines, nonlinear corrections, cosmic ray removal and correction for the tilt of the spectra (details may be found in Del Zanna et al. 2001a). A few minor corrections were applied to the GIS data, as described in Landi et al. (1999). Among the various detector effects, ghosting is one which can have a major impact on the validity of the derived intensity of an emission line. Although the GIS observations presented here were not badly affected by ghosts, some were present, and only in some of the cases was it possible to reconstruct the original spectra, i.e. add to each line the intensity lost by ghosting. In order to reduce the fixed patterning, the GIS spectra have been smoothed by applying a gaussian convolution. Line intensities were obtained by using multiple Gaussian line-fitting routines (see Haugan 1997) removing the "background" intensity, which is mostly detector-dependent.

The line intensities have been calibrated using the consistent NIS and GIS calibration of Del Zanna et al. (2001a), with an adjustement of the absolute values that takes into account the long term effects of the use of the NIS wide slit (W. Thompson, priv. comm.). The accuracy of the radiometric calibration of
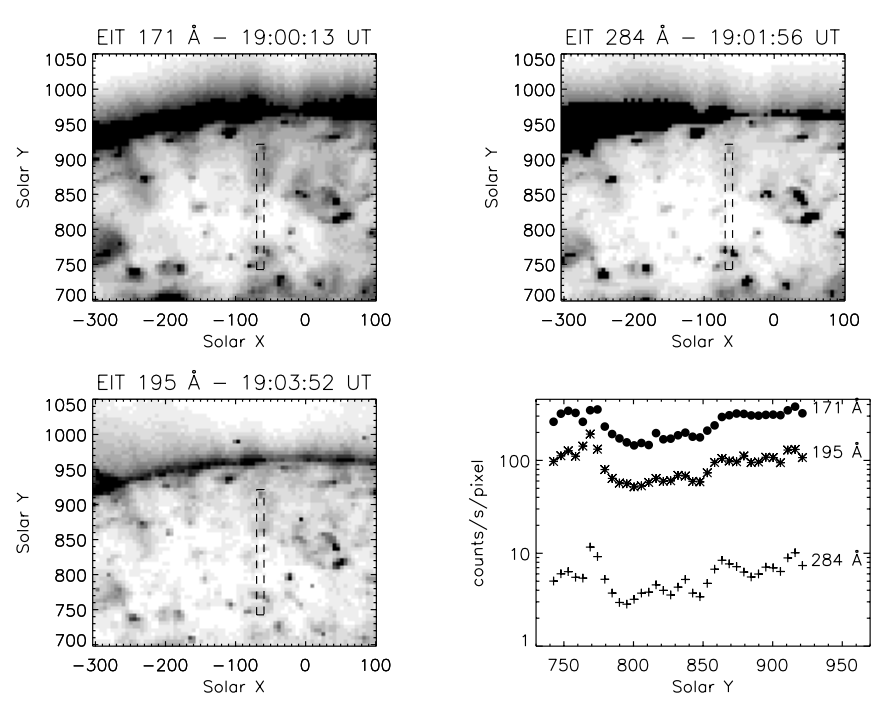

Fig. 3. EIT negative images - 23rd August 1996, showing the presence of a few polar plumes. That one observed by CDS was aligned almost $\mathrm{N}-\mathrm{S}$, at Solar $X=-70^{\prime \prime}$, with its base centre at about Solar $Y=850^{\prime \prime}$. The bottom right panel shows the signal in the three EIT filters along the region shown in the images.

the CDS detector can currently be estimated to be of the order of $30 \%$.

\subsection{The polar plume of 23 August 1996}

A Joint Operation Programme (JOP 48) was organised to observe this plume. During this programme, a number of $\mathrm{SOHO}$ instruments carried out simultaneous observations. Figure 3 shows the EIT images of this plume.

An analysis of the CDS observations has shown that this feature was stable during the whole observing period (a few hours). The NIS observations presented here contained a large selection of lines covering a wide range of temperatures. The area of the raster was $1^{\prime} \times 4^{\prime}$, formed by scanning with the $4^{\prime \prime} \times 240^{\prime \prime}$ slit, over a period of about an hour. Monochromatic images obtained from these data are shown in Fig. 4. This plume presents the typical characteristics of plumes as observed by CDS. The peak of the emission in the plume is seen in upper transition region lines such as Mg VII. It is less visible in the high-FIP Ne lines, and cannot be seen in the hightemperature (e.g. Fe XII) lines. Since the plume was aligned at an angle to the line of sight, the peak of the emission appears at different spatial locations when lines emitted at different temperatures are examined.

In order to increase the signal-to-noise ratio, averaged spectra of three regions, shown in Fig. 4, have been extracted: a profile along the plume, in the $\mathrm{N}-\mathrm{S}$ direction; a region at the base of the plume; and a coronal hole region, to the SW of the plume. Figure 5 shows the intensity ratios of some selected lines. The $\mathrm{O} / \mathrm{Ne}$ and $\mathrm{Mg} / \mathrm{Ne}$ ratios clearly show an enhancement in the region of the plume base at Solar $Y=850$, and also indicate a possible second plume in the line of sight at Solar $Y=890$. 

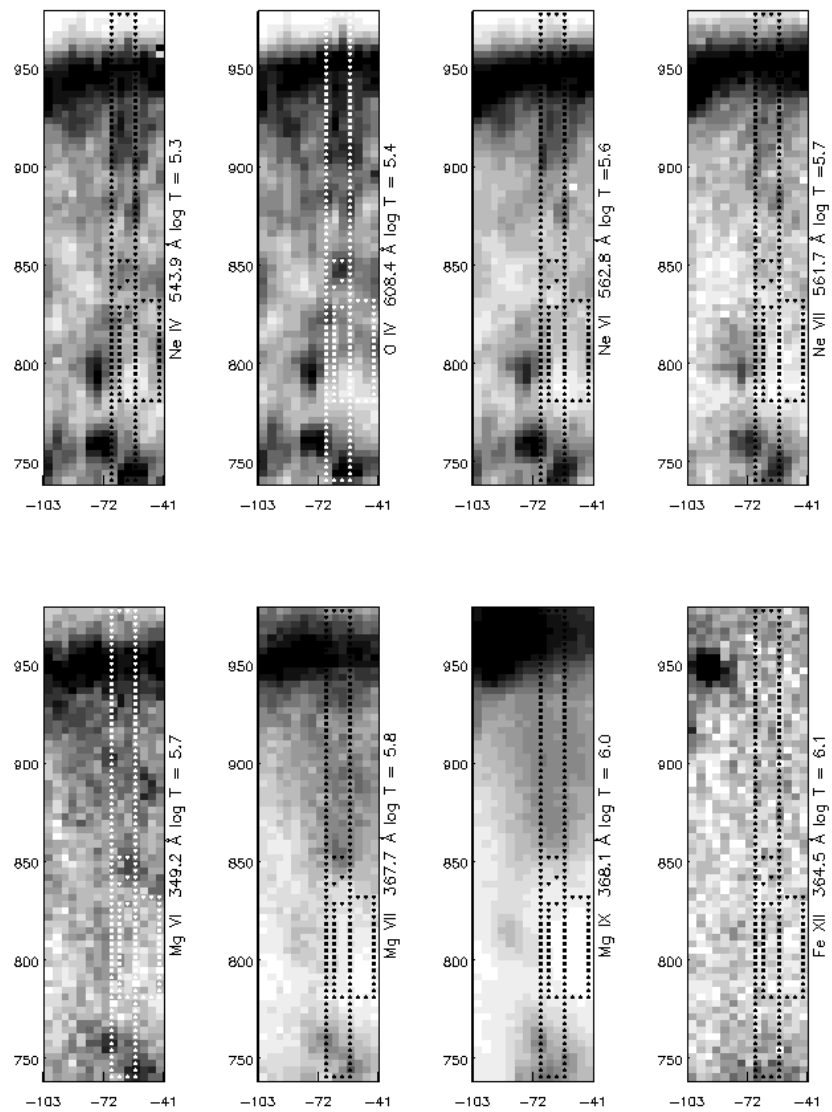

Fig. 4. Monochromatic images (negative) of the NIS observation 23rd August 1996. The plume is seen best in Mg VII and Mg IX, with its base near the centre of the raster and the "plume" extending up to the limb (and above). The three selected areas are shown (lower region: coronal hole; centre: base of the plume; profile along the N-S direction).

Table 1. Average electron densities $\left(\mathrm{cm}^{-3}\right)$ derived from the $23 \mathrm{rd}$ August 1996 spectra of the selected regions, plume base and coronal hole area.

\begin{tabular}{|c|c|c|}
\hline & Plume & C. hole \\
\hline$N_{\mathrm{e}}(\mathrm{O}$ IV $625.8 / 608.4 \AA)$ & $7.5 \pm 4 \times 10^{9}$ & $7.1 \pm 1 \times 10^{9}$ \\
\hline$N_{\mathrm{e}}(\mathrm{Mg}$ VIII 315.0/317.0 $\AA$ ) & $1.1 \pm 0.8 \times 10^{8}$ & $0.6 \pm 0.3 \times 10^{8}$ \\
\hline$N_{\mathrm{e}}(\mathrm{Mg}$ VIII $315.0 / 313.7 \AA)$ & $0.7 \pm 0.4 \times 10^{8}$ & $0.6 \pm 0.3 \times 10^{8}$ \\
\hline$N_{\mathrm{e}}(\mathrm{Si}$ IX 349.9/341.9 $\mathrm{A})$ & $1.1 \pm 0.7 \times 10^{8}$ & $0.5 \pm 0.5 \times 10^{8}$ \\
\hline$N_{\mathrm{e}}(\mathrm{Si}$ IX $349.9 / 345.1 \AA)$ & $0.9 \pm 0.5 \times 10^{8}$ & $0.3 \pm 0.3 \times 10^{8}$ \\
\hline
\end{tabular}

Figure 6 (lower plot) shows the average isothermal temperatures derived with the line ratio technique. Note that the three different line ratios produce different temperatures in the coronal hole region, while in the plume region they are in close agreement, another indication of the plumes bases being quasiisothermal, with lower temperatures $T \simeq 8 \times 10^{5} \mathrm{~K}$, rising slightly with distance along the plume. Note that these results are very different from those obtained with the EIT filter ratio technique (upper plot in Fig. 6), obtained with the SolarSoft routine eit_temp.pro. This routine, written by J. Newmark, uses a default set of parameters, the EIT effective areas and the
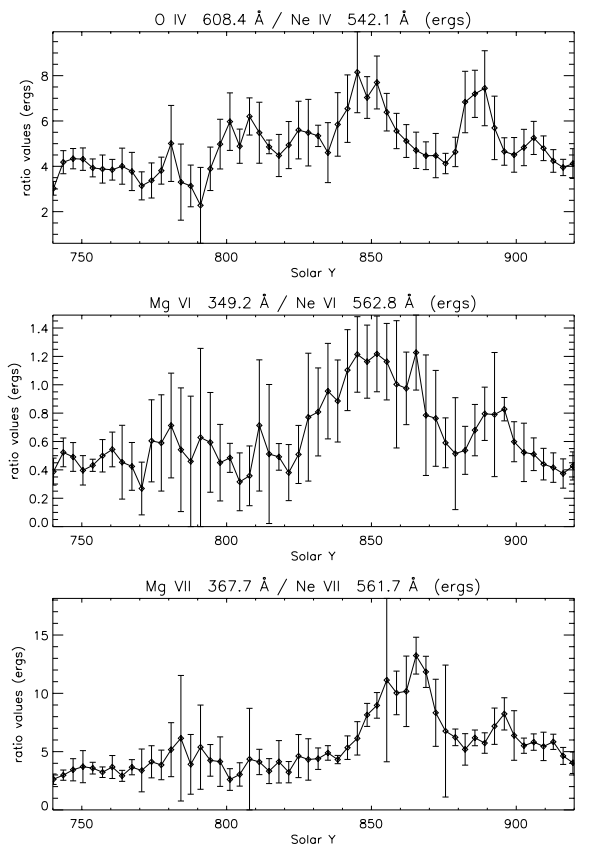

Fig. 5. Intensity ratios along the N-S direction - 23rd August 1996. The $\mathrm{O} / \mathrm{Ne}$ and $\mathrm{Mg} / \mathrm{Ne}$ ratios clearly show the presence of the plume base at Solar $Y=850$, and also indicate a possible second plume in the line of sight at Solar $Y=890$.

CHIANTI database to infer isothermal temperatures from the EIT count rates.

Table 1 shows the values of the densities deduced from the averaged spectra of the plume base and the nearby coronal hole region. Although the results have a relatively large scatter and large errors, the indication is that the density in the plume area was slightly higher than the nearby coronal hole region, which had a very low density, $N_{\mathrm{e}} \simeq 5 \times 10^{7} \mathrm{~cm}^{-3}$.

\subsection{The polar plume observations of $10 / 11$ October 1997}

Figure 7 displays a sequence of EIT $171 \AA$ images, showing the presence of a plume near the NW limb, and its stability over a period of few days. A series of CDS observations of this plume was performed during this period.

On 1997 October 10, a large $4^{\prime} \times 4^{\prime}$ region (see Fig. 8) was rastered three times with the NIS. The three rasters have been averaged, to increase the signal-to-noise (temporal variations within the two-hour observation were small). A few areas (see Fig. 8) have been selected for subsequent averaging of the spectra. They include: a coronal hole $(\mathrm{CH})$, the polar plume (PL), a region of quiet Sun (QS) and a bright point (BP) - the smallest region (see Del Zanna \& Bromage 1999c for details). The bright point was selected for comparison since from the EIT images it might be confused with a bright plume. Figure 9 shows the spectra of these regions for a few of the observed lines. The spectral characteristics of the various regions are quite different. The most striking feature is the large difference in the $\mathrm{Mg} \mathrm{VII} / \mathrm{Mg}$ IX ratio, clearly indicating the different temperature structure of the regions. The plume exhibits 

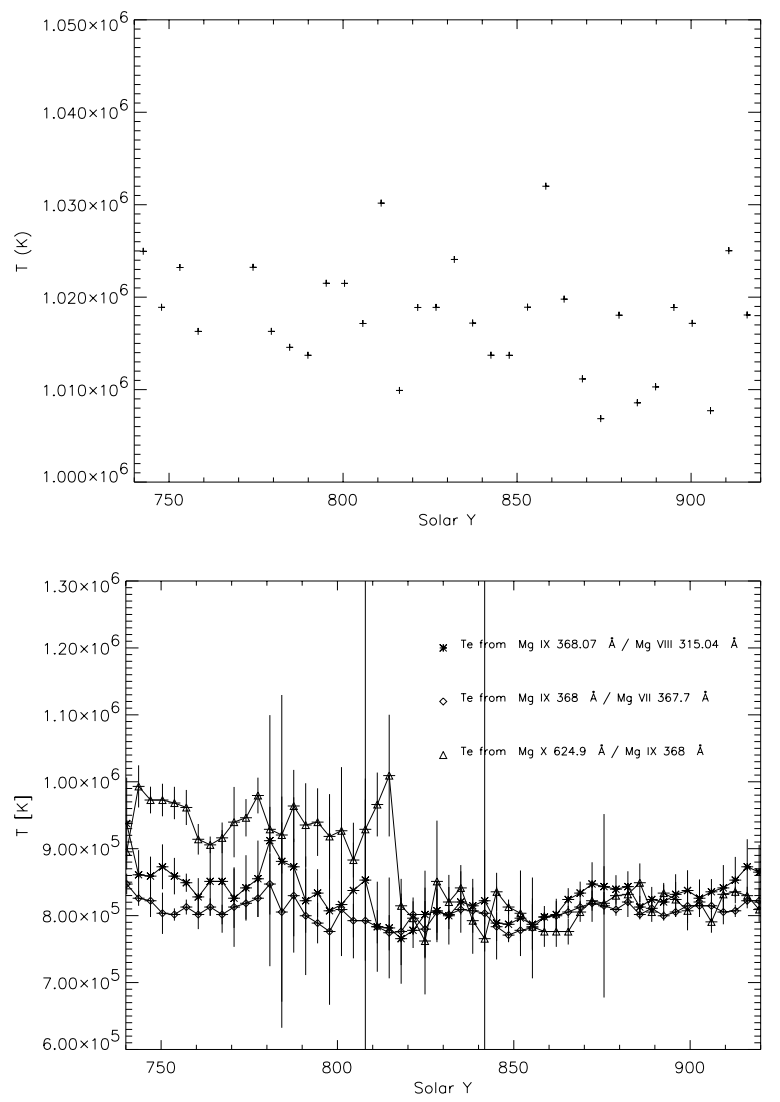

Fig. 6. Top: isothermal temperatures along the plume as derived from the EIT $195 \AA / 171 \AA$ Ailter ratio. Bottom: isothermal temperatures derived from CDS along the N-S profile - 23rd August 1996. Note that the plume lies along this direction, above about Solar $Y=825^{\prime \prime}$. This is the region where the temperature profiles become coincident, indicating isothermal plasma.

the same spectral characteristics as the Elephant's Trunk lowlatitude plume (Del Zanna \& Bromage 1999b).

Table 2. Table of parameters deduced from the 10th October 1997 averaged spectra of the selected regions. The densities, $\left(\mathrm{cm}^{-3}\right)$, are deduced from the following ratios: O IV [625.9 $\AA / 608.4 \AA]$; Mg VII [319.0 $\AA / 367.7 \AA]$; Si IX [349.9 $\AA / 341.9 \AA]$. The isothermal temperatures (in $10^{5} \mathrm{~K}$ ) are deduced from the $[\mathrm{Mg}$ IX $368.0 \AA / \mathrm{Mg}$ VII $367.7 \AA$ ก] ratio.

\begin{tabular}{lrrrr}
\hline \hline & PL & CH & QS & BP \\
\hline$N_{\text {e }}(\mathrm{O} \mathrm{IV})\left(10^{10}\right)$ & $0.7 \pm 0.1$ & $0.6 \pm 0.2$ & $1.3 \pm 0.3$ & $1.9 \pm 0.4$ \\
$N_{\mathrm{e}}\left(\mathrm{Mg}\right.$ VII) $\left(10^{9}\right)$ & $1.2 \pm 0.2$ & $0.5 \pm 0.2$ & $2.0 \pm 0.5$ & $0.9 \pm 0.5$ \\
$N_{\mathrm{e}}\left(\mathrm{Si} \mathrm{IX)}\left(10^{8}\right)\right.$ & $1.7 \pm 0.8$ & $1.4 \pm 0.9$ & $3.3 \pm 0.9$ & $5 \pm 2$ \\
$T_{\mathrm{e}}(\mathrm{Mg} \mathrm{X} / \mathrm{Mg}$ IX) & $8.1 \pm 0.1$ & $9.4 \pm 0.1$ & $11 \pm 1$ & $9.8 \pm 0.1$ \\
$T_{\mathrm{e}}(\mathrm{Mg} \mathrm{IX} / \mathrm{Mg}$ VII $)$ & $7.7 \pm 0.1$ & $8.2 \pm 0.1$ & $9.4 \pm 0.1$ & $8.6 \pm 0.1$ \\
\hline
\end{tabular}

Densities and temperatures of the various areas are presented in Table 2. Although the plume is not visible in the lower transition region line O IV, the density at its base can be measured and is found to be similar to the average coronal hole density, $N_{\mathrm{e}} \simeq 7 \times 10^{9} \mathrm{~cm}^{-3}$. In the quiet sun area, the density
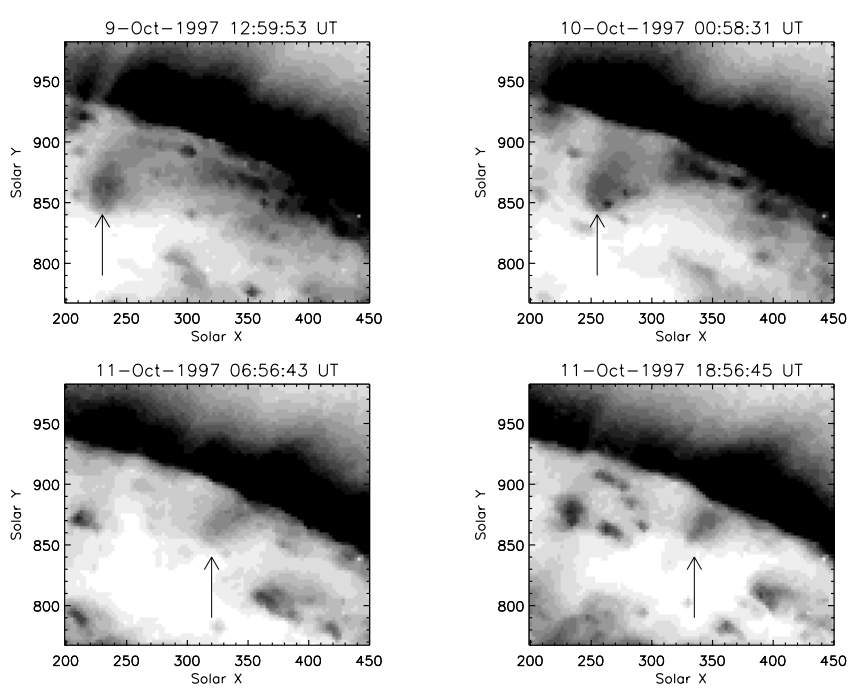

Fig. 7. Sequence of SOHO EIT $171 \AA$ negative images of the north polar coronal hole, showing the persistence of a polar plume for many days.
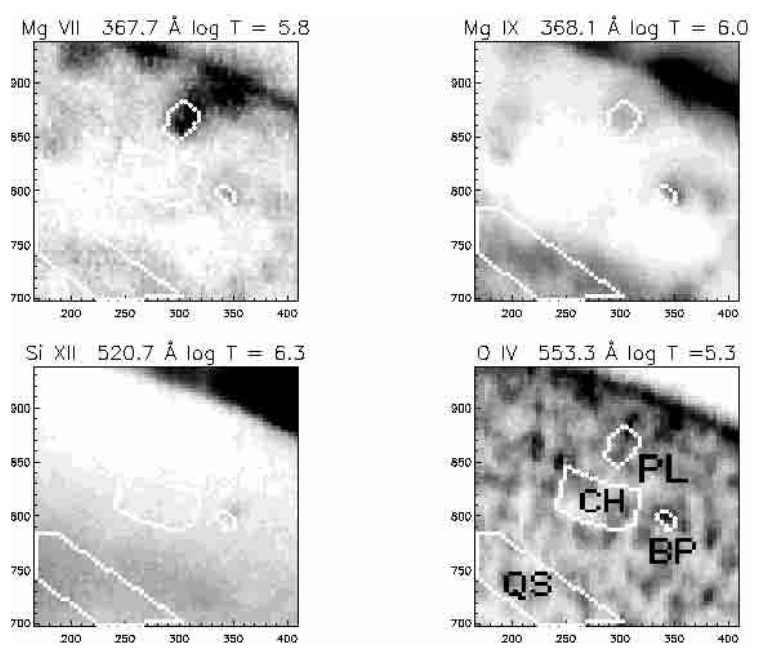

Fig. 8. Negative images in a few NIS lines - 10th October 1997, 18:2619:43 UT - with the selected areas indicated: plume (PL); bright point (BP); quiet sun (QS); coronal hole (CH).

derived from $\mathrm{O}$ IV is about 2 times higher than in the coronal hole region. At upper transition region temperatures, the plume was bright, so it was possible to derive an accurate density, $N_{\mathrm{e}}=1.2 \pm 0.2 \times 10^{9} \mathrm{~cm}^{-3}$, from the Mg VII $319 \AA$ line. Note that the quiet Sun region has a higher density, while the coronal hole area has a much lower density.

On the 11th October 1997, CDS was in high-telemetry mode (twice the usual rate, giving good temporal resolution), and special observing sequences were designed to observe the plume with both NIS and GIS. The NIS study included a large number of lines, this time scanning a $2^{\prime} \times 3^{\prime}$ area with the $4^{\prime \prime} \times 240^{\prime \prime}$ slit, from west to east. The NIS observation started at 15:00 UT. The GIS high-telemetry observations consisted of a west to east scan across the plume using the $4^{\prime \prime} \times 4^{\prime \prime}$ slit. At each of 15 positions, 100 exposures of $5 \mathrm{~s}$ each were taken, covering a $4^{\prime \prime} \times 60^{\prime \prime}$ region in a total time of nearly 3 hours. The 


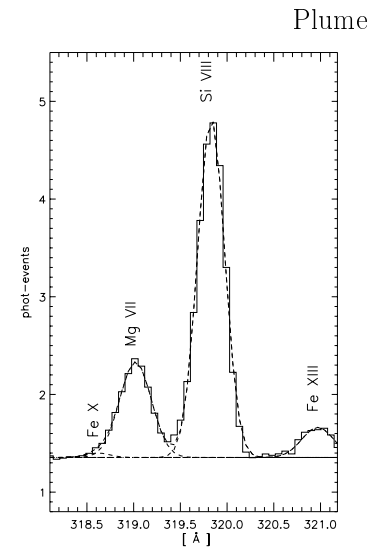

Plume - PL
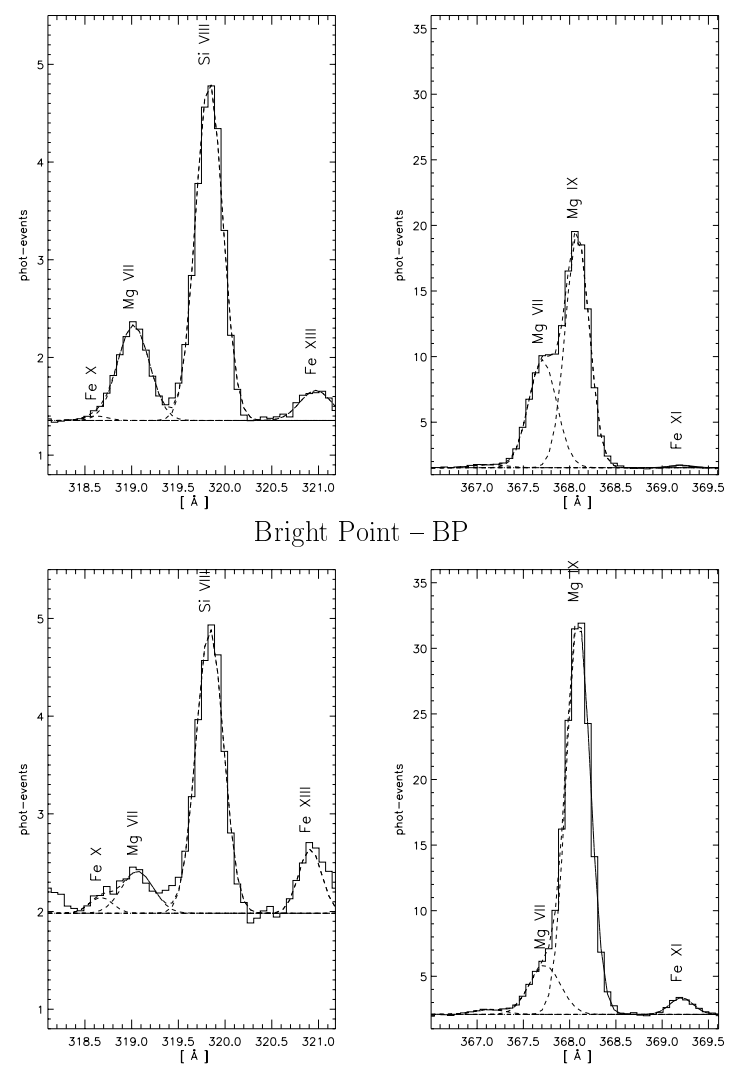

Quiet Sun - QS
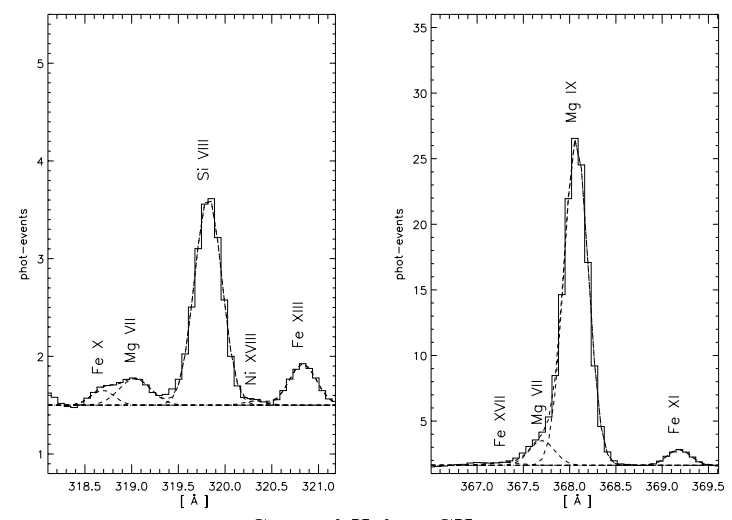

Coronal Hole - $\mathrm{CH}$
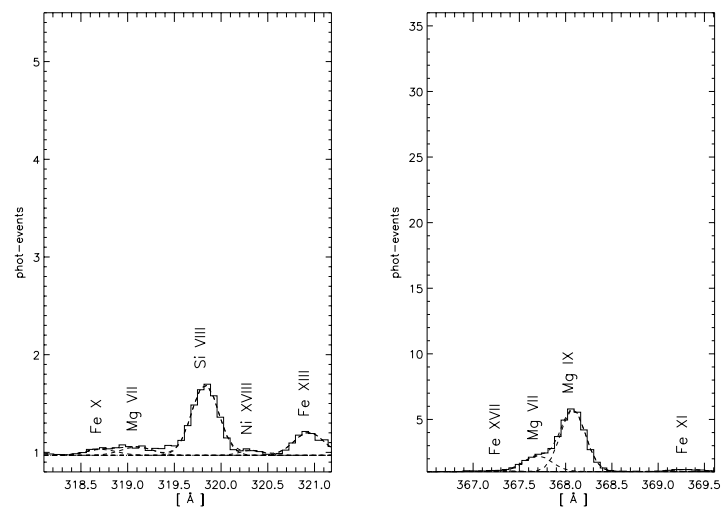

Fig. 9. Spectra in a few NIS windows of the selected areas - 10th October 1997. Note the large variations of the Mg VII/Mg IX ratio (right column). The Mg VII lines in these figures have been used for density measurements.
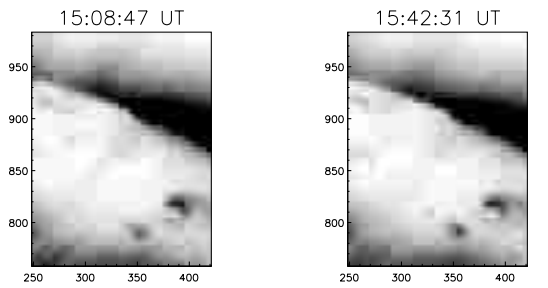

16:16:10 UT

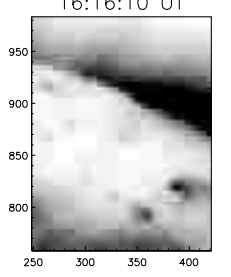

7:07:11 UT

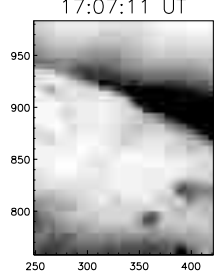

17:41:10 UT
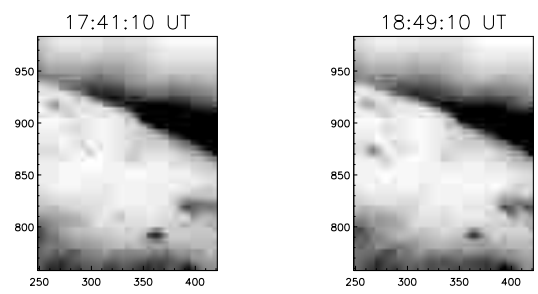

Fig. 10. EIT $195 \AA$ negative images of the plume during the times of the NIS and GIS observations - 11th October 1997. All images are plotted with the same intensity scale, showing that the plume has changed very little over this period.

GIS observations followed the NIS ones, starting at 16:05 UT and ending at 18:54 UT, so that the time between observing the plume itself with the two instruments was about two hours.

The October 11 observations are important because they include the first GIS observations of a plume. This allows the GIS data to be used to confirm the results (in particular the abundances) obtained using NIS data.

The stability of the plume during the time of the NIS and GIS observations was examined: Fig. 10 shows EIT images in the $195 \AA$ band spanning the times of the NIS and GIS observations of the plume. It shows very little variability in the intensity of this faint plume during this period, justifying the combination of the NIS and GIS data in the analysis. Small offsets between the recorded pointing of the EIT, NIS and GIS observations were found and corrected for. Direct comparisons of the line intensities also show good agreement (see Table A.2). A preliminary time analysis of the GIS observations confirmed that the intensity variations in the brightest lines were small. They were higher in the plume area (with indications of wave motions), but still within 20-30\%.

Figure 11 shows monochromatic images of the region rastered by the NIS. The intensities of the NIS lines have been determined from NIS spatially-averaged spectra over the region shown in Fig. 11, the same area rastered by the GIS. Figure 12 shows the intensity profiles of a sample of GIS and NIS lines. Figure 12 also shows how most of the lines have similar profiles (e.g. Mg IX, visible in both GIS and NIS) confirming the validity of the spatial alignment and the small time variability. Only the transition region lines present some differences, as 

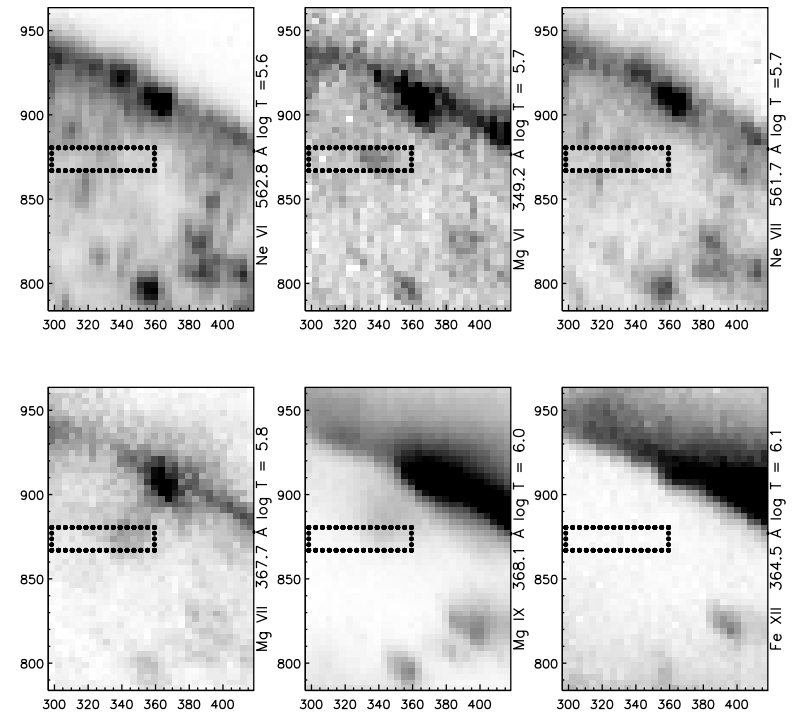

Fig. 11. Monochromatic images of the plume in a few NIS lines (negative) - 11th October 1997.
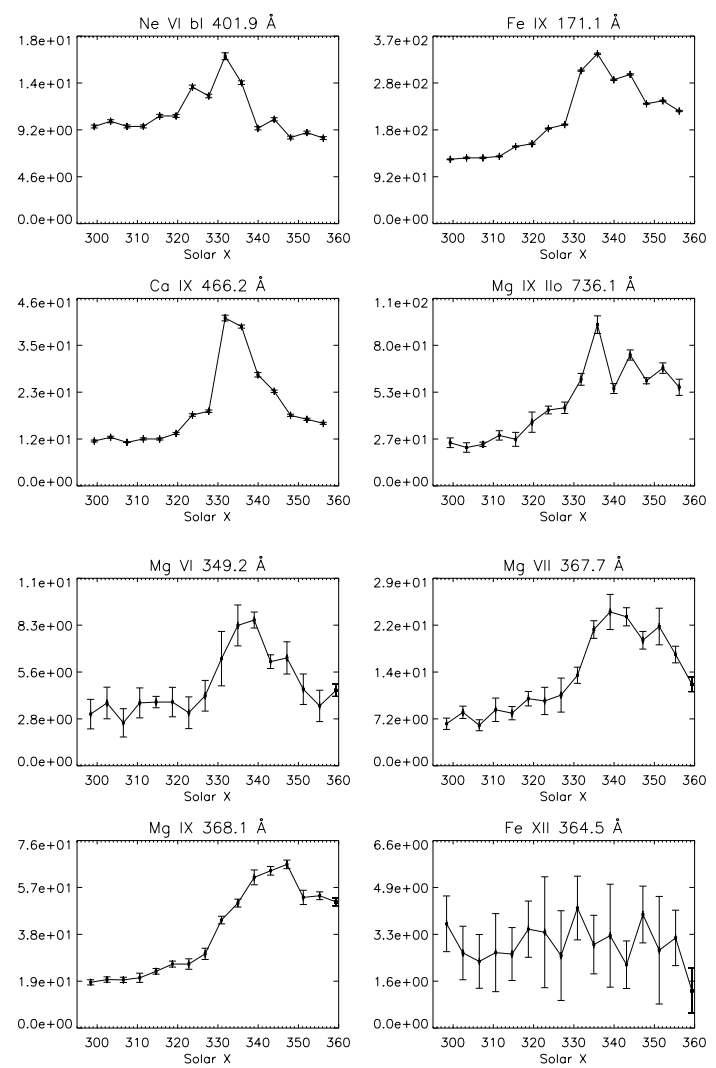

Fig. 12. Intensities of few selected GIS and NIS lines across the plume - 11th October 1997. Note that the intensities of the upper transition region lines increase by factors of 3-4 in the plume area.

expected. Figure 13 shows some intensity ratios derived from the GIS and NIS observation.

Inspection of Figs. 12 and 13 shows that this plume exhibits the same characteristics as both the polar plume of 23 August 1996, and the low-latitude plume in the Elephant's Trunk. In particular, it has maximum visibility in the upper transition
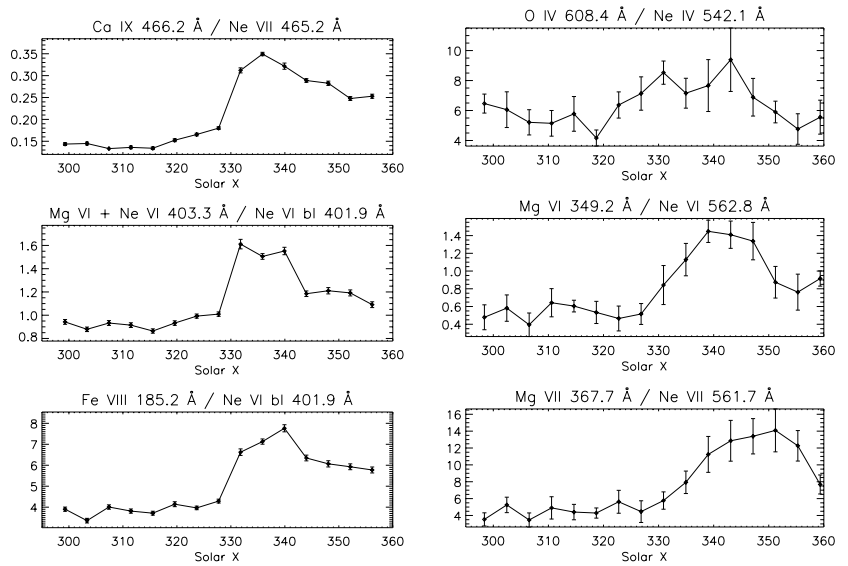

Fig. 13. Intensity ratios of few selected GIS and NIS lines across the plume - 11th October 1997. Note the large increase in the low- vs. high-FIP ratios.

Table 3. Table of densities (in $10^{8} \mathrm{~cm}^{-3}$ ) deduced from the October 11 1997 spectra of the selected regions.

\begin{tabular}{|c|c|c|}
\hline & Plume & C. hole \\
\hline$N_{\mathrm{e}}(\mathrm{Mg}$ VII $[319.0 \AA / 367.7 \AA])$ & $38 \pm 20$ & $7 \pm 7$ \\
\hline$N_{\mathrm{e}}(\mathrm{Mg}$ VIII $[315.0 \AA / 317.0 \AA ̊])$ & $0.7 \pm 0.3$ & $0.4 \pm 0.3$ \\
\hline$N_{\mathrm{e}}(\mathrm{Si}$ IX [349.9 ̊̊/341.9 ̊̊]) & $2.6 \pm 1$ & $0.6 \pm 0.3$ \\
\hline$N_{\mathrm{e}}(\mathrm{Si}$ IX $[349.9 \AA / 345.1 \AA])$ & $1.0 \pm 0.8$ & $0.8 \pm 0.5$ \\
\hline
\end{tabular}

region lines such as Mg VII, Ca X, Mg VIII (with an increase by a factor of about 4 ), while it is not seen in the high-FIP $\mathrm{Ne}$ lines, nor in high-temperature lines. The Mg VI/Ne VI, Ca IX/Ne VII Fe VIII/Ne VI ratios are clearly enhanced in the plume area. Although this is suggestive of a large FIP effect, it is shown in Sect. 3.3.1 below that this is not in fact the case.

Two spatial positions have been selected as representative of the coronal hole and plume areas, at Solar $X=298$ and 335, respectively. Figure 14 shows a comparison between the GIS spectra of the two areas, while Table 3 presents the densities.

The main features of the plume are: a large increase in the intensity of upper transition region lines (e.g. Mg VI, Mg VII, $\mathrm{Mg}$ VIII), with a small decrease in the high-temperature lines (e.g. Fe XV), and an enhancement of certain $\mathrm{Mg} / \mathrm{Ne}$ and $\mathrm{Ca} / \mathrm{Ne}$ ratios, as already discussed. In particular the regions around $402 \AA$ and $277 \AA$ show how different the spectra are. Other regions of the GIS spectra are shown in Figs. 19 and 20 (see Sect. 4, where the GIS-EIT comparison is presented). These also stress the different temperature structure between the coronal hole and the plume area examined. Lower temperature lines (such as Fe VIII and Fe IX) are greatly enhanced inside the plume.

\subsubsection{The DEM of the plume area}

A $D E M$ analysis of the combined intensities was performed, in order to better describe the thermal structure of the plume and to determine element abundances. A constant pressure of 

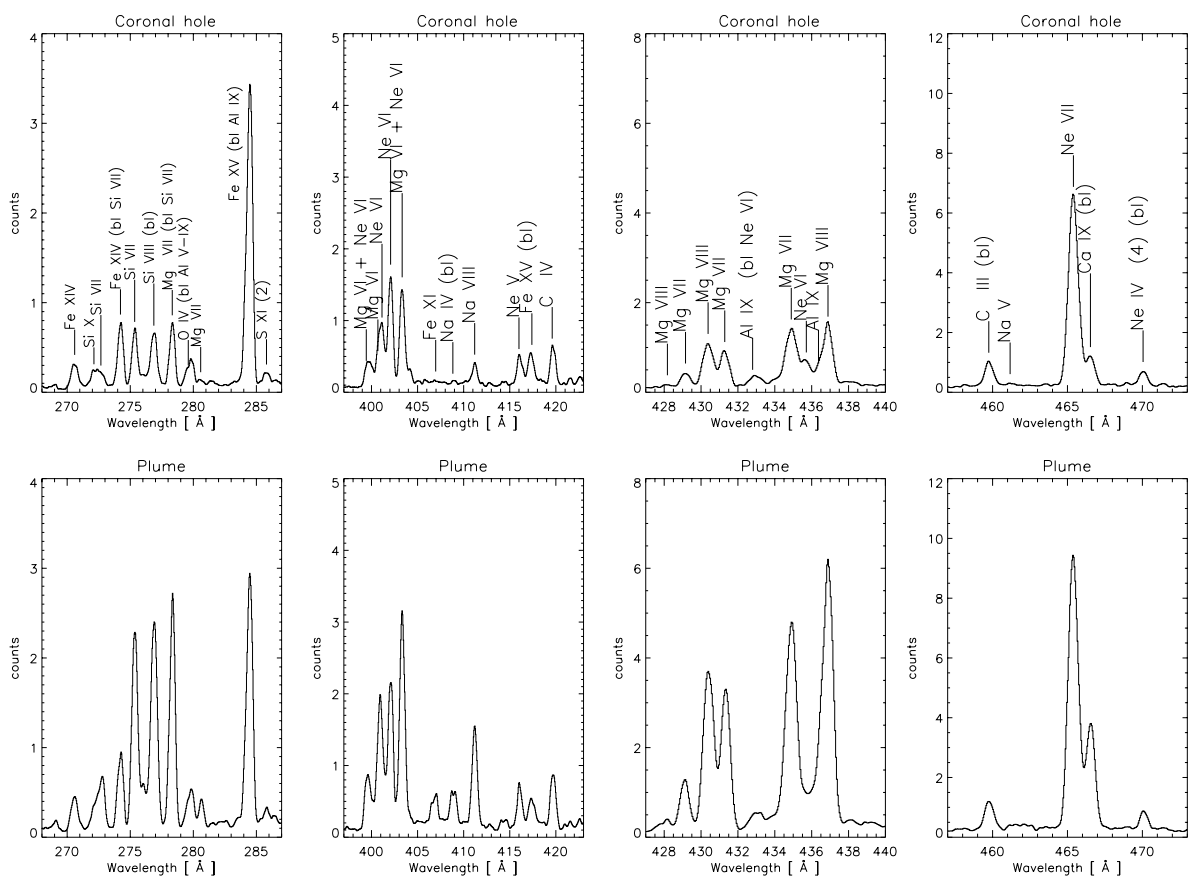

Fig. 14. GIS spectra in selected wavelength regions, for the coronal hole (above) and plume (below) areas - 11th October 1997.

$2 \times 10^{14} \mathrm{~cm}^{-3} \mathrm{~K}$ was adopted when calculating the contribution functions. As a starting point, we adopted the photospheric abundances of Grevesse and Sauval (1998), with a correction of 0.1 dex in the oxygen abundance, as suggested by recent measurements (N. Grevesse, priv. comm.).

The results are shown in Fig. 16 and in Table A.2. The DEM peaks at upper transition region temperatures (Mg VII, Ca X), $T=7.9 \times 10^{5} \mathrm{~K}$ with a quasi-isothermal distribution at these heights. Table A. 2 shows that there is good agreement between most of the NIS and GIS lines, at least when lines close in temperature are considered. It should be noted that the lines from the Li-like ions are underestimated or overestimated by large factors (as already discussed), and are not shown. Moreover, a number of other important ions such as Fe VIII presented similar problems and are also not shown.

The result is that of all the elements, only the Ne abundance needed to be changed (lowered by $0.2 \mathrm{dex}$ ). This is well constrained at low transition region temperatures, where bright O III, Ne III, O IV, Ne IV, O V, Ne V lines are observed. This result appears quite surprising, and deserves a more detailed examination in the future. We only note here that there are many other reported cases of $\mathrm{Ne}$ abundance variations (compared to both high and low FIP elements, see Schmelz et al. 1996) in different solar structures, but none for coronal plumes.

In order to confirm the $D E M$ results of this plume, we have performed the same DEM analysis on the 23 August plume and on the Elephant's trunk low-latitude plume. Figure 17 shows the $D E M$ of the latter. In both cases, similar results were found; i.e. a $D E M$ peaking at $T=7.9 \times 10^{5} \mathrm{~K}$, and photospheric abundances, with the exception of $\mathrm{Ne}$, where a lower (by about a 0.2 dex) abundance was required.
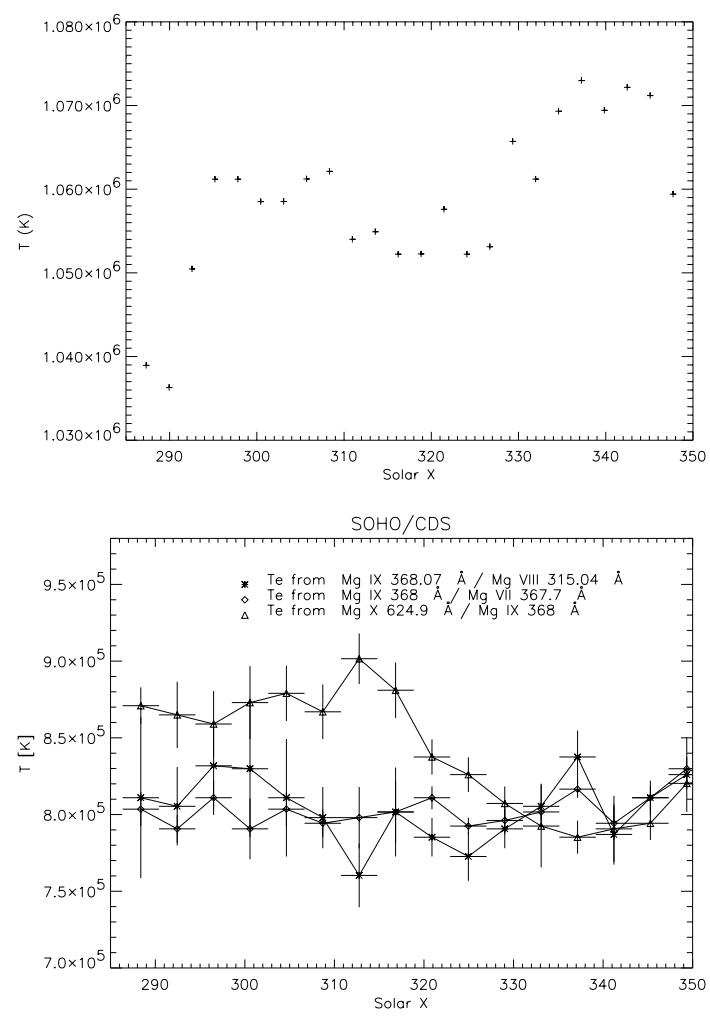

Fig. 15. Isothermal temperatures as derived from the EIT $195 \AA$ / $171 \AA$ filter ratio (above, see Fig. 18 for the extraction region) and CDS lines (below). The CDS line ratios indicate isothermality $\left(T_{\mathrm{e}} \simeq 8 \times 10^{5} \mathrm{~K}\right)$ in the plume region. Note that the plume $D E M$ peaks at $T_{\mathrm{e}} \simeq 7.9 \times 10^{5} \mathrm{~K}$.

\section{Comparison of GIS spectra with EIT images}

The EIT instrument (Delaboudiniere et al. 1995) observes the Sun in four EUV filters, centred around 171, 195, 284, and 


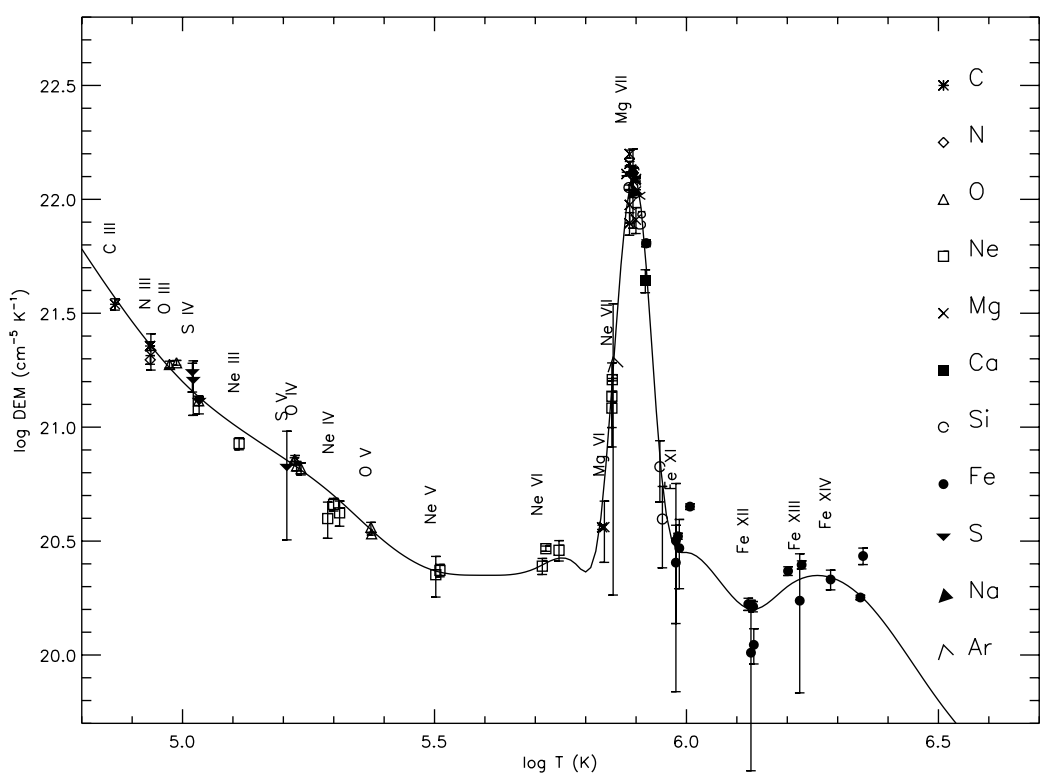

Fig. 16. The DEM distribution of the plume area (solid line) with the observed points. Each experimental data point is plotted at the effective temperature $T_{\text {eff }}$ and at a value equal to the product $D E M\left(T_{\text {eff }}\right) \times\left(I_{\mathrm{ob}} / I_{\mathrm{th}}\right)$. The error bars refer to the combination of observational and theoretical $(10 \%)$ errors and give an idea of the uncertainties in the derived $D E M$ values.

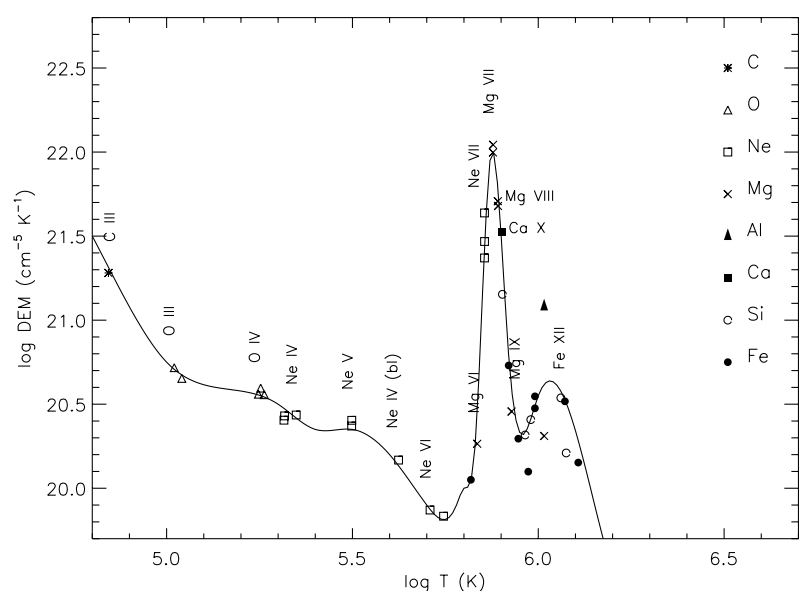

Fig. 17. The DEM distribution of the Elephant's trunk equatorial plume, showing similar characteristics to that one in Fig. 16. The Ne abundance was lowered by $0.25 \mathrm{dex}$, compared to the photospheric value.

$303 \AA$. The first two are the principal ones, since the filter at $284 \AA$ has a lower sensitivity, and the filter at $303 \AA$ includes in the bandpass both the chromospheric line He II $303.8 \AA$ and the coronal line Si XI 303.3 $\AA$. The first two filters are often referred to as the Fe IX/X and the Fe XII images, because it is thought that these are the ions that contribute most to the emission. Ratios of these filters have been used widely to derive temperatures. In particular, DeForest et al. (1997) used the EIT filter-ratio method to show that plumes are about $30 \%$ cooler than the surrounding coronal hole regions.

Here we test the validity of such an approach by applying the EIT ratio technique to the plume observed with GIS, which was described above in Sect. 3. A comparison between the GIS scan and the EIT $171 \AA$ and $195 \AA$ images was performed, using full-resolution EIT images taken just after the GIS spectra. A direct comparison is possible because GIS spectrally resolves the emission lines observed by the EIT filters. Average intensity profiles in the two EIT filters, over the region observed by GIS are shown in Fig. 18.

The observed and calibrated GIS spectra were then convolved with the EIT effective areas (Dere et al. 2000), to simulate the observed EIT count rates, at each spatial position. The count rates were then integrated over the passband, and the results are shown in Fig. 18. The agreement between the observed and simulated EIT counts is quite good, in particular for the 171 and $195 \AA$ filters, considering all the uncertainties in the absolute calibrations of both EIT and GIS.

On the other hand, no true diagnostic ratio can be inferred from the EIT count rates. For example, the real Fe XII $195.1 \AA / \mathrm{Fe}$ IX $171.07 \AA$ ratio as measured by GIS is lower than the value of the corresponding EIT filter ratio at the plume base, and higher in the coronal hole region. In terms of temperatures, we have already given two examples above (Figs. 6 and $15)$ where the results inferred from the EIT on-disk measurements are quite different (both in terms of absolute and relative values) from those that are directly measured by CDS.

To illustrate in more detail how the observed EIT intensity changes depending on the type of solar structure observed, GIS spectra of the two previously selected regions, chosen as representative of coronal hole and plume areas, are plotted in Figs. 19-21 with the same spectra multiplied by the EIT filter response. A quiet sun GIS observation has been added for comparison. Although the three spectra presented should not be regarded as canonic spectra of such solar features, they do show how different lines, formed at different temperatures, contribute to the emission seen by the EIT filters. Considering first the EIT $171 \AA$ filter, Fig. 19 shows that in the coronal hole and plume regions the main intensity is from $\mathrm{Fe}$ IX and Fe X lines, with Fe IX dominating, while in the quiet sun spectrum the Fe IX $171 \AA$ becomes weaker and Fe X is the main contributor. The EIT $171 \AA$ A filter therefore records in any of these 

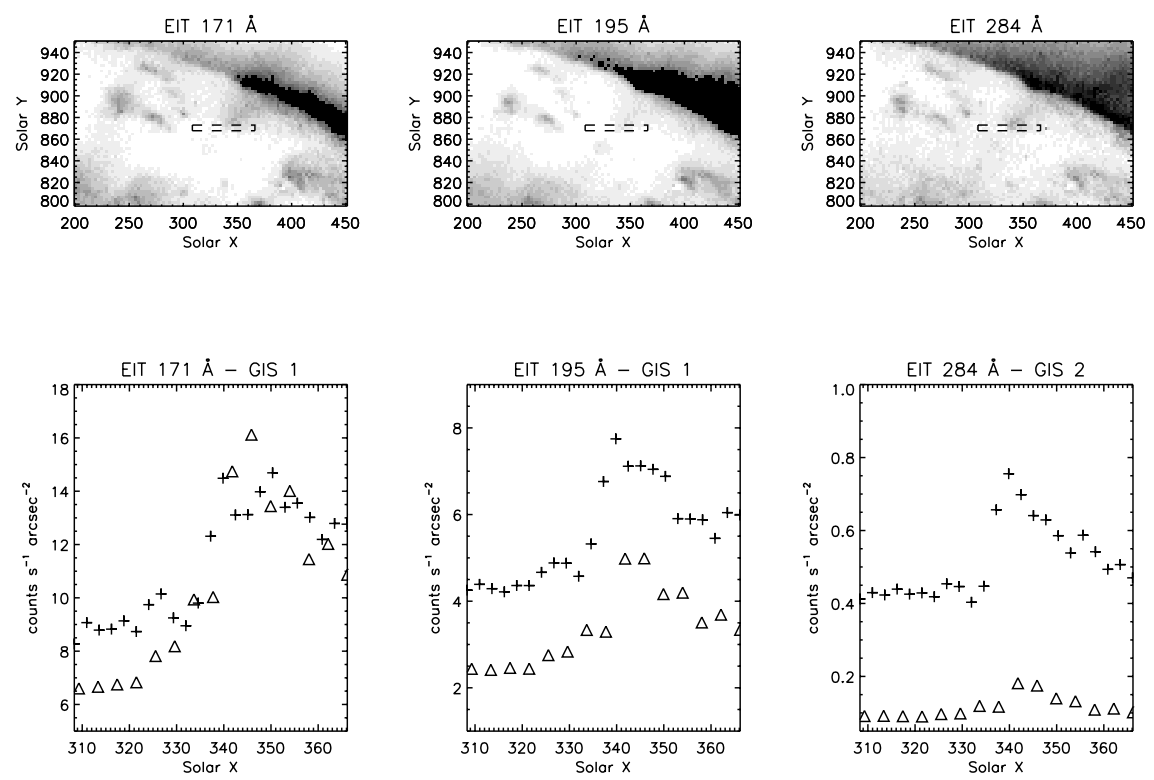

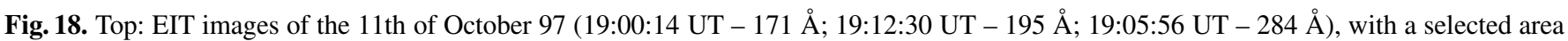
across the plume overlaid. Bottom: intensity profiles of the selected area in EIT $171 \AA$ (crosses) with superimposed the observed CDS intensity filtered (triangles). The bottom figures indicate problems in the EIT/GIS cross-calibration for the 195 and $284 \AA$ A filters.
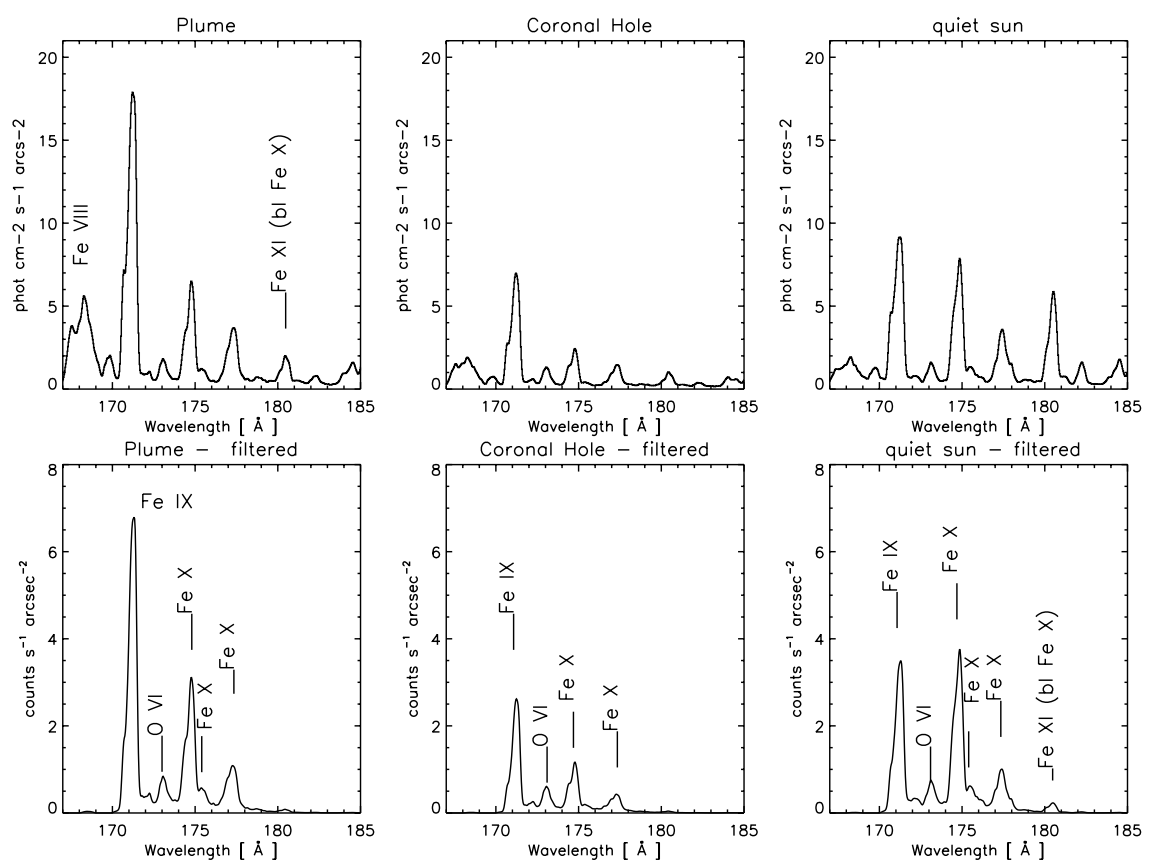

Fig. 19. Top: GIS 1 spectra of plume, coronal hole and quiet sun. Bottom: same as above, but multiplied by the EIT $171 \AA ̊$ filter response. The EIT is dominated by Fe IX and Fe X lines, while the Fe VIII lines, very strong in the plume, are not observed by the EIT filter. Note, however, that any small error in the EIT passband can have a considerable effect.

three cases plasma emission produced by a restricted temperature range, and can be regarded as almost isothermal, although a weak O VI line complicates the picture.

In the EIT $195 \AA$ filter, the situation is not quite the same, as Fig. 20 shows. While in the quiet sun spectrum the main contribution is from Fe XII lines, mixed with some $\mathrm{Fe} \mathrm{X}, \mathrm{Fe} \mathrm{XI}$ and Fe XIII emission, in the coronal hole and plume spectra the cooler lines (Fe VIII, Fe X, Fe XI) become increasingly important, compared to the Fe XII lines, as one would expect. This shows that it is incorrect to assume that the observed emission in the EIT $195 \AA$ filter is predominantly from Fe XII, and explains why plumes are visible in the EIT $195 \AA$ images. Furthermore, since the lines observed in the EIT $195 \AA$ filter are formed over a wide range of temperatures, it is strictly inappropriate to use this filter for temperature diagnostics. 

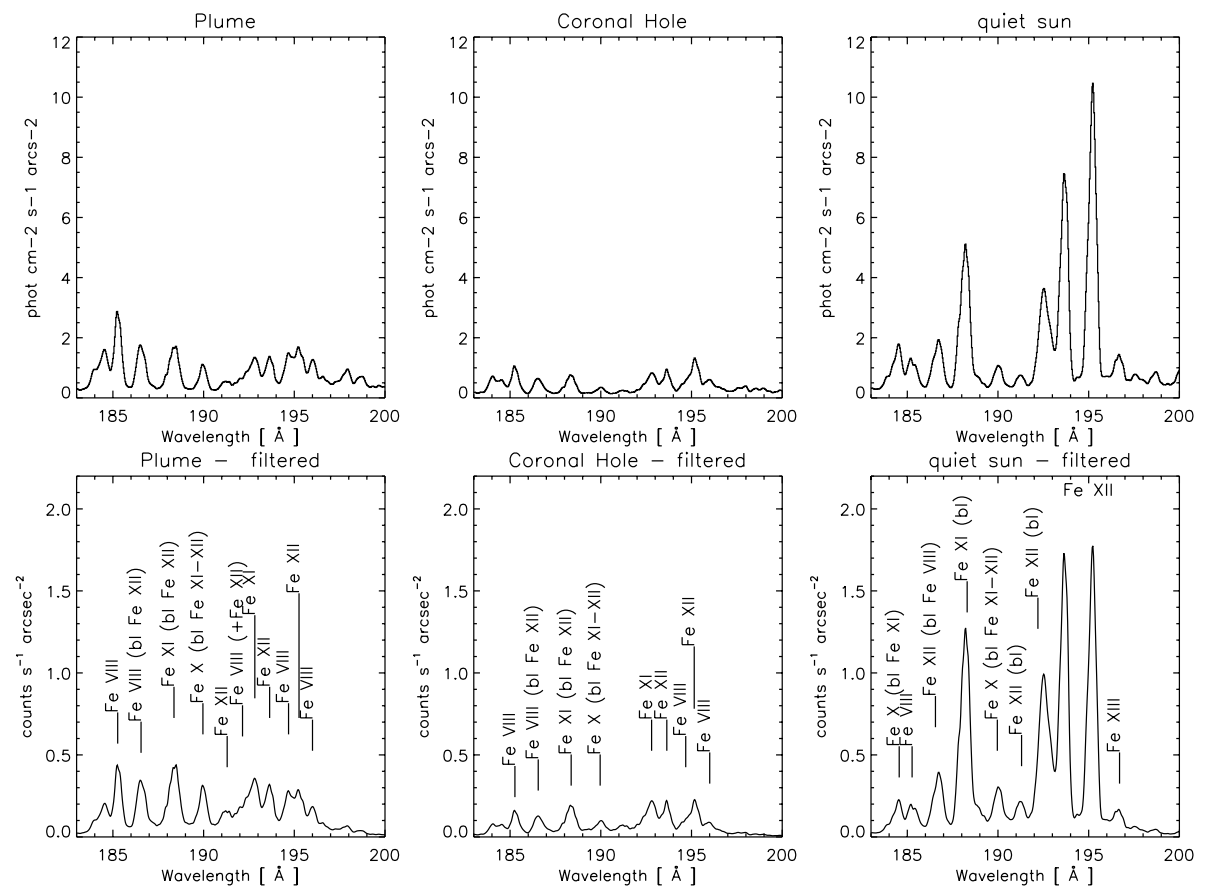

Fig. 20. Top: GIS 1 spectra of plume, coronal hole and quiet sun. Bottom: same as above, but multiplied by the EIT $195 \AA$ A filter response. Note that while in the quiet Sun the EIT signal is dominated by Fe XII lines, in the coronal hole lower temperature lines become significant. In the plume, the signal is dominated by Fe VIII lines. The 195 Å filter is therefore not isothermal.

It is therefore not surpising that the isothermal temperatures derived from the EIT filters produce incorrect results. A similar result applies to the EIT $284 \AA$ filter, that is not isothermal. In fact, the emission is a mix of Fe XV and much cooler lines (Si VII, Mg VII), as Fig. 21 shows. This lack of isothermality, together with an offset in the absolute calibration (see Fig. 18) results in the fact that the combination of the $284 \AA$ filter with the $195 \AA$ one produces much higher (by a factor of about 2) temperatures than the $171 / 195 \AA$ ratio.

In order to look for an explanation to the high temperatures derived from the EIT filter ratios, we have calculated the EIT temperatures by assuming photospheric abundances, a pressure of $1 \times 10^{14} \mathrm{~cm}^{-3} \mathrm{~K}$ and the latest version 4 of the CHIANTI database, but the results did not change significantly. We have also applied a correction to the absolute values of the EIT effective areas to match the observed GIS intensities with the EIT count rates. This correction improves the results, in the sense that the temperatures derived from the two EIT filter ratios become lower, but still higher then the values obtained spectroscopically. A rigorous GIS/EIT cross-calibration is in progress (in collaboration with J. Newmark) which might lead to the possibility of inferring more reliable temperatures from EIT images. We note that any filter ratio technique should include a $D E M$ analysis and checks on the consistency between all the EIT filters.

\section{Summary and conclusions}

CDS spectroscopic observations made on the solar disk have been used to characterise polar plumes, comparing them with earlier observations of a low-latitude plume, seen in the Elephant's Trunk coronal hole near disk-centre (Del Zanna \& Bromage 1999b). These plumes are all diffuse, quasi-stable structures, rather than the category of plume which has a bright, hot footpoint. The latter may in fact be an earlier stage in the evolution of the plume. Here, we have presented the results from two plumes, typical of the many such diffuse structures to be seen in the polar coronal holes around solar minimum. They exhibit the same characteristic signature as the low-latitude plume, namely, enhancement of the cool, upper transition region lines such as $\mathrm{Mg}$ VII, no emission in the hotter coronal lines (formed above $1.2 \times 10^{6} \mathrm{~K}$ ) and an order-ofmagnitude increase in ratios of high-FIP to low-FIP lines (e.g. $\mathrm{Mg}$ VII/Ne VII).

We have presented the first GIS observations of the base of a plume, and used them to confirm the results obtained from NIS observations. In particular, we have used them to better define the $D E M$ and the elemental abundances. We have shown that the $D E M$ technique, combined with the large number of observed lines and a good instrument calibration, is extremely useful for obtaining information on relative element abundances and on the temperature distribution along the line of sight. We have shown that other methods, widely used, can lead to inaccurate results. However, the results of the $D E M$ analysis depend critically on the ionization fractions adopted. We have pointed out that the currently available ionization equilibrium calculations for a significant number of ions lead to large inconsistencies. Unfortunately, it has been common practice to use lines from ions with anomalous behaviour for diagnostic studies of solar and stellar atmospheres. This has had serious consequences in the derivation of densities and element 

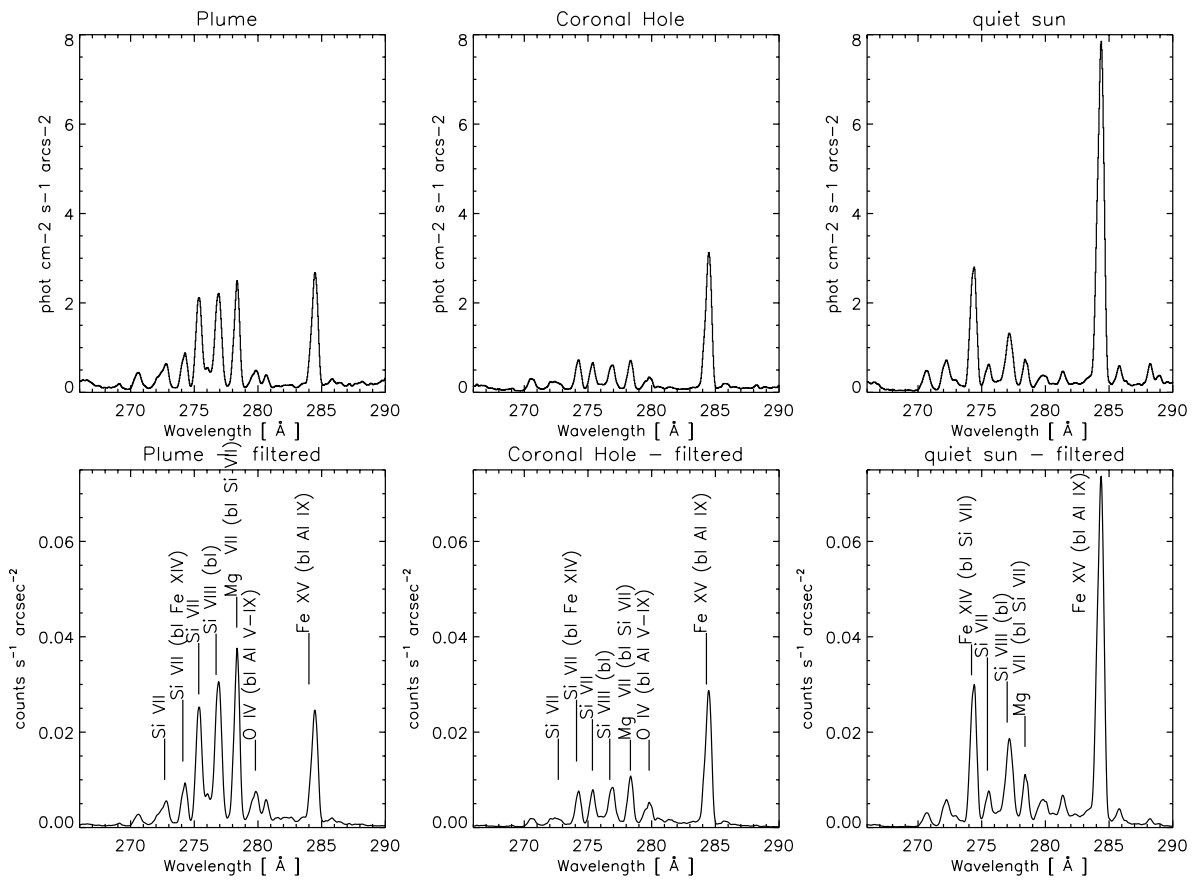

Fig. 21. Top: GIS 3 spectra of plume, coronal hole and quiet sun. Bottom: same as above, but multiplied by the EIT $284 \AA$ filter response. Note that it is only in the quiet Sun that the EIT signal is dominated by Fe XV, while in the plume base the main contributions are from lines that are formed at much lower temperatures. The $284 \AA$ filter is therefore not isothermal.

abundances from stellar EUV spectra as shown by Del Zanna et al. (2002). Any previous results on the solar atmosphere based on the use of these ions should therefore be taken with caution. More refined emission measure and element abundance studies will only be possible when the problems in the ionization equilibria have been solved.

Both line ratio techniques and $D E M$ analysis have shown plume plasma to be close to isothermal, with a temperature $T \simeq$ $7-8 \times 10^{5} \mathrm{~K}$, a little cooler than the surrounding coronal hole region. This was found using a number of different line-ratios and was also the temperature at which a sharp peak in emission measure can be seen in the $D E M$ curve. The less isothermal emission from the surrounding coronal hole region can be seen as a broader peak in the $D E M$, close to, or just below, $1 \times 10^{6} \mathrm{~K}$.

For any theoretical modelling, it would be important to study in detail any temperature variations across and along the length of the plumes. The observations presented here indicate a small increase in temperature with height along the plumes and no variation across them. A more detailed study will be the subject of a future paper. Here we only mention that Del Zanna (1999) has shown that plumes have shallower radial temperature gradients compared to the inter-plume regions, and that these temperature differences tend to disappear with distance, as all the coronal hole plasma becomes almost isothermal.

Accurate density measurements are difficult to obtain, because of the weakness of the density-sensitive lines. At lowercoronal heights $\left(T \simeq 8-9 \times 10^{5} \mathrm{~K}\right.$; e.g., Mg VIII, Si IX) they have rarely shown significant differences between plume and inter-plume regions $\left(N_{\mathrm{e}} \simeq 2 \times 10^{8} \mathrm{~cm}^{-3}\right)$. However, due to low signal, the uncertainties are large and do not exclude the possibility of slightly higher density in plumes. At lower temperatures $\left(T \sim 7 \times 10^{5} \mathrm{~K}\right)$, where plumes have their maximum emission measure and visibility, the density (Mg VII) is $N_{\mathrm{e}} \simeq 1.2 \times 10^{9}\left(\mathrm{~cm}^{-3}\right)$, higher than in the coronal hole regions, but similar to quiet sun values. At even lower temperatures $\left(T \sim 2 \times 10^{5} \mathrm{~K}\right)$, in the lower transition region (O IV), plumes are not visible, but O IV emission at the base of the plume indicates densities which are the same as in the nearby coronal hole network regions $\left(N_{\mathrm{e}} \simeq 5 \times 10^{9} \mathrm{~cm}^{-3}\right)$.

It has been shown that the characteristic line intensities in the plume are mainly a result of their quasi-isothermal temperature structure. In particular, the large enhancement of some intensity ratios (such as $\mathrm{Mg} \mathrm{VII} / \mathrm{Ne} \mathrm{VII}$ ) seen at the base of the plumes can largely be explained by this temperature effect, rather than implying large changes in abundance.

We have not found any strong evidence for an FIP effect in plumes, contrary to earlier reports. We have shown that the Skylab plume observations can similarly be explained by the plume's temperature characteristics. However, in the plumes we observed, we have found a consistent small reduction in Ne abundance (relative to photospheric values). The result that plumes do not after all exhibit any significant FIP effect means that the previous deduction (see the introduction), that plumes could not be the source of the fast solar wind, is no longer valid. However, our result does not prove that they are the source of the fast wind. Indeed, as described in the introduction, other recent studies suggest that the source region is more likely to be between the plumes.

We have used the plume GIS observations to show that the EIT filter response is not isothermal. In particular, we have shown that, in the case of plumes, the low-temperature lines provide the dominant contribution to the EIT filter images. As 
a result, these filters cannot be reliably used for temperature estimates without an in-depth analysis. We note that similar arguments apply to other broad-band filter ratio techniques such as those based on Transition Region And Coronal Explorer (TRACE) data, that have been widely used in the last few years. This is because TRACE filters have similar responses to the EIT ones. All the above shows the importance of complementing any intensity measurements with spectroscopic observations in order to determine the physical parameters of various solar coronal features. Care must also be taken that the analysis technique adopted is appropriate for the conditions of the observation.

Acknowledgements. G. Del Zanna and H.E. Mason acknowledge support from PPARC. The authors thank Jeff Newmark for providing EIT images and effective areas, and the CDS team for support in the observations. GDZ was also supported by a University of Central Lancashire studentship for part of this work. SOHO is a project of international collaboration between ESA and NASA.

\section{Appendix A:}

Table A.1. Results on the Skylab plume. The columns consist of: (1) the ion; (2) the theoretical wavelength $\lambda_{\text {th }}$; (3) the observ $\lambda_{\mathrm{ob}}$; (4) configuration and term description; (5) observed intensity $I_{\mathrm{ob}}\left(\mathrm{ergs} \mathrm{cm}^{-2} \mathrm{~s}^{-1} \mathrm{sr}^{-1}\right)$; (6) ratio between the theoretical $I_{\mathrm{th}}$ an intensity; (7) the estimated error on the ratio value; (8) the effective temperature $T_{\text {eff }}\left(\log _{10}\right.$ values); (9) $T_{\max }\left(\log _{10}\right.$ values); (10) tl of each transition to the total calculated intensity of the line.

\begin{tabular}{|c|c|c|c|c|c|c|c|c|c|}
\hline Ion & $\begin{array}{l}\lambda_{\text {th }} \\
(\AA)\end{array}$ & $\begin{array}{l}\lambda_{\mathrm{ob}} \\
(\AA ̊)\end{array}$ & Transition & $I_{\mathrm{ob}}$ & $\begin{array}{l}I_{\mathrm{th}} / \\
I_{\mathrm{ob}}\end{array}$ & $+/-$ & $\begin{array}{l}\log \\
T_{\text {eff }}\end{array}$ & $\begin{array}{l}\log \\
T_{\max }\end{array}$ & $\%$ \\
\hline $\mathrm{Ne}$ VI & 401.928 & 401.94 & $2 \mathrm{~s}^{2} 2 \mathrm{p}^{2} \mathrm{P}_{3 / 2}-2 \mathrm{~s} 2 \mathrm{p}^{2}{ }^{2} \mathrm{P}_{3 / 2}$ & 35.9 & 1.00 & 0.14 & 5.87 & 5.62 & \\
\hline $\mathrm{Ne}$ VI & 399.826 & 399.83 & $2 \mathrm{~s}^{2} 2 \mathrm{p}^{2} \mathrm{P}_{1 / 2}-2 \mathrm{~s} 2 \mathrm{p}^{2}{ }^{2} \mathrm{P}_{3 / 2}$ & 6.8 & 1.04 & 0.15 & 5.87 & 5.62 & \\
\hline $\mathrm{Ne}$ VI & 401.154 & 401.14 & $2 \mathrm{~s}^{2} 2 \mathrm{p}^{2} \mathrm{P}_{1 / 2}-2 \mathrm{~s} 2 \mathrm{p}^{2}{ }^{2} \mathrm{P}_{1 / 2}$ & 9.6 & 1.50 & 0.80 & 5.88 & 5.62 & \\
\hline Mg VI & 403.307 & 403.32 & $2 \mathrm{~s} 2.2 \mathrm{p} 3{ }^{4} \mathrm{~S}_{3 / 2}-2 \mathrm{~s} .2 \mathrm{p} 4{ }^{4} \mathrm{P}_{5 / 2}$ & 70.9 & 1.03 & 0.15 & 5.89 & 5.66 & \\
\hline Mg VI & 399.281 & 399.27 & $2 \mathrm{~s} 2.2 \mathrm{p} 3{ }^{4} \mathrm{~S}_{3 / 2}-2 \mathrm{~s} .2 \mathrm{p} 4{ }^{4} \mathrm{P}_{1 / 2}$ & 37.6 & 0.65 & 0.09 & 5.89 & 5.66 & \\
\hline Mg VI & 400.662 & 400.68 & $2 \mathrm{~s} 2.2 \mathrm{p} 3{ }^{4} \mathrm{~S}_{3 / 2}-2 \mathrm{~s} .2 \mathrm{p} 4{ }^{4} \mathrm{P}_{3 / 2}$ & 46.7 & 1.06 & 0.15 & 5.89 & 5.66 & \\
\hline $\mathrm{Ne}$ VII & 465.220 & 465.20 & $2 \mathrm{~s} 2{ }^{1} \mathrm{~S}_{0}-2 \mathrm{~s} 2 \mathrm{p}{ }^{1} \mathrm{P}_{1}$ & 127.0 & 5.76 & 0.81 & 5.89 & 5.71 & \\
\hline Mg VII & 434.906 & 434.80 & $2 s^{2} 2 p^{2}{ }^{3} P_{2}-2 s 2 p^{3}{ }^{3} D_{3}$ & 168.0 & 0.78 & 0.11 & 5.90 & 5.81 & 0.87 \\
\hline Mg VII & 434.708 & & $2 \mathrm{~s}^{2} 2 \mathrm{p}^{2}{ }^{3} \mathrm{P}_{2}-2 \mathrm{~s} 2 \mathrm{p}^{3}{ }^{3} \mathrm{D}_{2}$ & & & & & 5.81 & 0.13 \\
\hline Mg VII & 431.305 & 431.25 & $2 \mathrm{~s}^{2} 2 \mathrm{p}^{2}{ }^{3} \mathrm{P}_{1}-2 \mathrm{~s} 2 \mathrm{p}^{3}{ }^{3} \mathrm{D}_{2}$ & 163.0 & 0.53 & 0.08 & 5.90 & 5.81 & 0.78 \\
\hline Mg VII & 431.191 & & $2 \mathrm{~s}^{2} 2 \mathrm{p}^{2}{ }^{3} \mathrm{P}_{1}-2 \mathrm{~s} 2 \mathrm{p}^{3}{ }^{3} \mathrm{D}_{1}$ & & & & & 5.81 & 0.22 \\
\hline Ca IX & 466.239 & 466.23 & $3 \mathrm{~s} 2{ }^{1} \mathrm{~S}_{0}-3 \mathrm{~s} .3 \mathrm{p}{ }^{1} \mathrm{P}_{1}$ & 58.2 & 1.06 & 0.15 & 5.90 & 5.80 & \\
\hline $\mathrm{Na}$ VIII & 411.166 & 411.17 & $2 \mathrm{~s} 2{ }^{1} \mathrm{~S}_{0}-2 \mathrm{~s} .2 \mathrm{p}{ }^{1} \mathrm{P}_{1}$ & 81.6 & 0.78 & 0.11 & 5.91 & 5.86 & \\
\hline Mg VIII & 436.734 & 436.70 & $2 s^{2} 2 p^{2} P_{3 / 2}-2 s 2 p^{2}{ }^{2} D_{5 / 2}$ & 253.0 & 1.15 & 0.16 & 5.92 & 5.90 & \\
\hline Mg VIII & 430.455 & 430.47 & $2 s^{2} 2 p^{2} P_{1 / 2}-2 s 2 p^{2}{ }^{2} D_{3 / 2}$ & 137.0 & 1.18 & 0.17 & 5.92 & 5.90 & \\
\hline $\mathrm{Ca} X$ & 574.010 & 574.02 & $3 s^{2} S_{1 / 2}-3 p^{2} P_{1 / 2}$ & 21.4 & 0.95 & 0.13 & 5.97 & 5.86 & \\
\hline $\mathrm{CaX}$ & 557.765 & 557.75 & $3 s^{2} S_{1 / 2}-3 p{ }^{2} P_{3 / 2}$ & 46.8 & 0.88 & 0.12 & 5.97 & 5.86 & \\
\hline
\end{tabular}


Table A.2. Results for the 1997 October 11 CDS observation of a polar coronal hole plume. The columns consist of: (1) the ion; (2) the theoretical wavelength $\lambda_{\mathrm{th}}$; (3) the observed wavelength $\lambda_{\mathrm{ob}}$; (4) observed intensity $I_{\mathrm{ob}}\left(\mathrm{ergs} \mathrm{cm} \mathrm{s} \mathrm{s}^{-1} \mathrm{sr}^{-1}\right.$ ); (5) the CDS detector and channel (G: GIS; N: NIS); (6) ratio between the theoretical $I_{\text {th }}$ and the observed intensity; (7) the estimated error on the ratio value; (8) the effective temperature $T_{\text {eff }}\left(\log _{10}\right.$ values); (9) $T_{\max }\left(\log _{10}\right.$ values); (10) the contribution of each transition to the total calculated intensity of the line.

\begin{tabular}{|c|c|c|c|c|c|c|c|c|c|}
\hline Ion & $\begin{array}{l}\lambda_{\text {th }} \\
(\AA)\end{array}$ & $\begin{array}{l}\lambda_{\mathrm{ob}} \\
(\AA)\end{array}$ & $I_{\mathrm{ob}}$ & $\begin{array}{l}I_{\mathrm{th}} / \\
I_{\mathrm{ob}}\end{array}$ & $+/-$ & Det & $\begin{array}{l}\log \\
T_{\text {eff }}\end{array}$ & $\begin{array}{l}\log \\
T_{\text {max }}\end{array}$ & $\%$ \\
\hline$\overline{\mathrm{C}}$ III & 538.313 & 538.3 & 35.2 & 1.07 & 0.12 & N 2 & 4.87 & 4.96 & 0.39 \\
\hline O II & 538.320 & & & & & & & 4.86 & 0.27 \\
\hline C III & 538.150 & & & & & & & 4.96 & 0.23 \\
\hline C III & 459.628 & 459.8 & 23.1 & 1.01 & 0.11 & G 3 & 4.94 & 4.97 & 0.46 \\
\hline C III & 459.515 & & & & & & & 4.97 & 0.25 \\
\hline C III & 459.467 & & & & & & & 4.97 & 0.11 \\
\hline N III & 685.816 & 685.8 & 64.2 & 1.16 & 0.13 & G 4 & 4.94 & 4.98 & 0.72 \\
\hline N III & 685.515 & & & & & & & 4.98 & 0.28 \\
\hline N III & 684.998 & 684.8 & 10.2 & 1.05 & 0.22 & G 4 & 4.94 & 4.98 & \\
\hline O III & 702.895 & 702.7 & 83.8 & 0.97 & 0.10 & G 4 & 4.97 & 5.01 & 0.39 \\
\hline O III & 702.821 & & & & & & & 5.01 & 0.30 \\
\hline O III & 702.890 & & & & & & & 5.01 & 0.24 \\
\hline O III & 703.850 & 703.7 & 146.1 & 0.88 & 0.09 & G 4 & 4.99 & 5.01 & 0.75 \\
\hline O III & 703.845 & & & & & & & 5.01 & 0.25 \\
\hline S IV & 748.393 & 748.9 & 11.7 & 0.86 & 0.15 & G 4 & 5.02 & 5.06 & \\
\hline S IV & 753.760 & 753.9 & 5.8 & 0.93 & 0.27 & G 4 & 5.02 & 5.06 & \\
\hline O III & 525.808 & 525.9 & 43.5 & 1.04 & 0.12 & $\mathrm{~N} 2$ & 5.03 & 5.05 & \\
\hline S IV & 661.396 & 661.8 & 26.8 & 1.09 & 0.14 & G 4 & 5.03 & 5.07 & 0.90 \\
\hline S IV & 661.455 & & & & & & & 5.06 & 0.10 \\
\hline $\mathrm{Ne}$ III & 489.495 & 489.1 & 18.2 & 1.17 & 0.14 & G 3 & 5.11 & 5.06 & 0.47 \\
\hline $\mathrm{Ne}$ III & 488.093 & & & & & & & 5.06 & 0.16 \\
\hline $\mathrm{Ne}$ III & 488.851 & & & & & & & 5.06 & 0.13 \\
\hline $\mathrm{Ne}$ III & 490.295 & & & & & & & 5.06 & 0.12 \\
\hline S V & 663.126 & 663.3 & 3.1 & 1.12 & 0.57 & G 4 & 5.21 & 5.21 & \\
\hline O IV & 554.521 & 554.6 & 290.5 & 0.94 & 0.10 & $\mathrm{~N} 2$ & 5.22 & 5.23 & \\
\hline O IV & 555.264 & 555.4 & 59.5 & 0.94 & 0.10 & $\mathrm{~N} 2$ & 5.22 & 5.23 & \\
\hline O IV & 553.337 & 553.4 & 55.0 & 0.99 & 0.11 & $\mathrm{~N} 2$ & 5.23 & 5.23 & \\
\hline O IV & 608.399 & 608.4 & 42.8 & 0.99 & 0.12 & N 2 & 5.24 & 5.23 & \\
\hline $\mathrm{Ne}$ IV & 542.071 & 542.2 & 6.0 & 1.33 & 0.27 & $\mathrm{~N} 2$ & 5.29 & 5.29 & \\
\hline $\mathrm{Ne} I V$ & 469.831 & 470.1 & 12.4 & 1.10 & 0.13 & G 3 & 5.30 & 5.31 & 0.58 \\
\hline $\mathrm{Ne} I V$ & 469.875 & & & & & & & 5.31 & 0.38 \\
\hline $\mathrm{Ne} I V$ & 543.887 & 544.0 & 10.8 & 1.13 & 0.18 & $\mathrm{~N} 2$ & 5.31 & 5.29 & \\
\hline $\mathrm{OV}$ & 760.444 & 760.5 & 40.0 & 0.98 & 0.11 & G 4 & 5.37 & 5.38 & 0.84 \\
\hline $\mathrm{O} \mathrm{V}$ & 760.225 & & & & & & & 5.38 & 0.16 \\
\hline $\mathrm{OV}$ & 629.730 & 629.8 & 662.9 & 1.04 & 0.10 & $\mathrm{~N} 2$ & 5.38 & 5.38 & \\
\hline $\mathrm{Ne} \mathrm{V}$ & 569.837 & 569.9 & 5.0 & 1.04 & 0.23 & $\mathrm{~N} 2$ & 5.50 & 5.48 & 0.76 \\
\hline $\mathrm{Ne} \mathrm{V}$ & 569.759 & & & & & & & 5.48 & 0.24 \\
\hline $\mathrm{Ne} \mathrm{V}$ & 416.210 & 416.1 & 9.3 & 0.98 & 0.12 & G 3 & 5.51 & 5.48 & \\
\hline $\mathrm{Ne} \mathrm{VI}$ & 562.798 & 562.9 & 20.4 & 1.01 & 0.13 & N 2 & 5.71 & 5.62 & \\
\hline $\mathrm{Ne} \mathrm{VI}$ & 401.928 & 402.1 & 33.3 & 0.86 & 0.09 & G 3 & 5.72 & 5.62 & \\
\hline $\mathrm{Ne} \mathrm{VI}$ & 558.592 & 558.7 & 14.7 & 0.92 & 0.13 & $\mathrm{~N} 2$ & 5.75 & 5.62 & 0.78 \\
\hline $\mathrm{Ne}$ VII & 558.609 & & & & & & & 5.71 & 0.22 \\
\hline Mg VI & 403.307 & 403.3 & 50.0 & 1.24 & 0.13 & G 3 & 5.83 & 5.67 & 0.90 \\
\hline $\mathrm{Ne} \mathrm{VI}$ & 403.270 & & & & & & & 5.62 & 0.10 \\
\hline $\mathrm{Mg}$ VI & 349.163 & 349.2 & 23.0 & 1.56 & 0.49 & N 1 & 5.84 & 5.66 & 0.52 \\
\hline Mg VI & 349.124 & & & & & & & 5.66 & 0.39 \\
\hline
\end{tabular}


Table A.2. continued.

\begin{tabular}{|c|c|c|c|c|c|c|c|c|c|}
\hline Ion & $\begin{array}{l}\lambda_{\text {th }} \\
(\AA)\end{array}$ & $\begin{array}{l}\lambda_{\mathrm{ob}} \\
(\AA)\end{array}$ & $I_{\mathrm{ob}}$ & $\begin{array}{c}I_{\mathrm{th}} / \\
I_{\mathrm{ob}}\end{array}$ & $+/-$ & Det & $\begin{array}{l}\log \\
T_{\text {eff }}\end{array}$ & $\begin{array}{l}\log \\
T_{\max }\end{array}$ & $\%$ \\
\hline$\overline{\mathrm{Ne}} \mathrm{VII}$ & 564.528 & 564.7 & 2.5 & 1.10 & 0.45 & N2 & 5.85 & 5.71 & \\
\hline $\mathrm{Ne}$ VII & 561.728 & 561.7 & 6.9 & 1.24 & 0.25 & $\mathrm{~N} 2$ & 5.85 & 5.71 & \\
\hline $\mathrm{Ne}$ VII & 465.220 & 465.4 & 232.7 & 0.98 & 0.10 & G 3 & 5.85 & 5.71 & \\
\hline Ar VIII & 519.440 & 519.2 & 0.3 & 0.98 & 0.89 & $\mathrm{~N} 2$ & 5.85 & 5.62 & \\
\hline Mg VII & 434.906 & 434.9 & 101.9 & 0.71 & 0.07 & G 3 & 5.88 & 5.81 & 0.85 \\
\hline Mg VII & 434.708 & & & & & & & 5.81 & 0.13 \\
\hline Si VII & 275.353 & 275.4 & 103.8 & 0.98 & 0.10 & G 2 & 5.89 & 5.78 & \\
\hline Mg VII & 367.659 & 367.7 & 55.0 & 1.45 & 0.22 & N 1 & 5.89 & 5.81 & 0.76 \\
\hline Mg VII & 367.672 & & & & & & & 5.81 & 0.24 \\
\hline Mg VII & 431.305 & 431.3 & 61.0 & 0.80 & 0.08 & G 3 & 5.89 & 5.81 & 0.78 \\
\hline Mg VII & 431.191 & & & & & & & 5.81 & 0.22 \\
\hline Mg VII & 429.123 & 429.1 & 22.8 & 0.73 & 0.08 & G 3 & 5.89 & 5.81 & \\
\hline Mg VII & 365.221 & 365.2 & 39.5 & 1.22 & 0.24 & N 1 & 5.89 & 5.81 & 0.37 \\
\hline Mg VII & 365.163 & & & & & & & 5.81 & 0.35 \\
\hline Mg VII & 365.235 & & & & & & & 5.81 & 0.27 \\
\hline Mg VII & 278.393 & 278.3 & 106.2 & 0.91 & 0.09 & G 2 & 5.89 & 5.82 & 0.66 \\
\hline Si VII & 278.443 & & & & & & & 5.78 & 0.33 \\
\hline $\mathrm{Na}$ VIII & 411.166 & 411.2 & 25.9 & 1.22 & 0.13 & G 3 & 5.89 & 5.86 & \\
\hline Mg VII & 363.750 & 363.8 & 14.6 & 1.07 & 0.42 & N 1 & 5.89 & 5.81 & \\
\hline $\mathrm{Fe} I X$ & 171.073 & 171.1 & 1891.3 & 0.99 & 0.10 & G 1 & 5.89 & 5.85 & \\
\hline Si VIII & 277.057 & 276.9 & 120.6 & 0.97 & 0.10 & G 2 & 5.90 & 5.92 & 0.36 \\
\hline Mg VII & 276.993 & & & & & & & 5.82 & 0.33 \\
\hline Si VII & 276.839 & & & & & & & 5.78 & 0.14 \\
\hline Si VIII & 276.850 & & & & & & & 5.90 & 0.14 \\
\hline Mg VIII & 436.734 & 436.9 & 121.1 & 1.05 & 0.11 & G 3 & 5.90 & 5.90 & 0.89 \\
\hline Mg VIII & 436.661 & & & & & & & 5.91 & 0.11 \\
\hline Mg VIII & 315.016 & 315.2 & 172.6 & 1.17 & 0.12 & G 2 & 5.90 & 5.90 & \\
\hline Mg VIII & 315.016 & 315.0 & 129.7 & 1.55 & 0.26 & N 1 & 5.90 & 5.90 & \\
\hline Mg VIII & 430.455 & 430.4 & 80.9 & 1.02 & 0.10 & G 3 & 5.90 & 5.91 & \\
\hline $\mathrm{Ca} \mathrm{X}$ & 557.765 & 557.8 & 12.8 & 1.29 & 0.20 & N 2 & 5.92 & 5.86 & \\
\hline $\mathrm{Fe} X$ & 184.543 & 184.5 & 129.3 & 0.80 & 0.08 & G 1 & 5.92 & 5.99 & \\
\hline $\mathrm{Fe} X$ & 174.534 & 174.7 & 623.2 & 0.77 & 0.08 & G 1 & 5.92 & 5.99 & \\
\hline Si IX & 345.124 & 345.1 & 26.5 & 1.10 & 0.35 & N 1 & 5.95 & 6.06 & 0.71 \\
\hline Si IX & 344.951 & & & & & & & 6.07 & 0.24 \\
\hline Si IX & 349.873 & 349.8 & 15.5 & 1.43 & 0.57 & N 1 & 5.95 & 6.04 & 0.82 \\
\hline Si IX & 349.794 & & & & & & & 6.06 & 0.18 \\
\hline Fe XI & 352.662 & 352.7 & 12.1 & 1.15 & 0.54 & N 1 & 5.98 & 6.06 & \\
\hline $\mathrm{Fe} X \mathrm{XI}$ & 369.153 & 369.3 & 4.5 & 0.92 & 0.73 & N 1 & 5.98 & 6.06 & \\
\hline $\mathrm{Fe} X \mathrm{XI}$ & 180.408 & 180.5 & 205.1 & 0.86 & 0.09 & G 1 & 5.98 & 6.07 & 0.79 \\
\hline $\mathrm{Fe} X$ & 180.407 & & & & & & & 5.99 & 0.17 \\
\hline Fe XII & 352.106 & 352.1 & 19.9 & 0.96 & 0.34 & $\mathrm{~N} 1$ & 5.99 & 6.14 & 0.43 \\
\hline Al VII & 352.159 & & & & & & & 5.82 & 0.32 \\
\hline $\mathrm{Na}$ VII & 352.302 & & & & & & & 5.78 & 0.12 \\
\hline Mg V & 352.201 & & & & & & & 5.47 & 0.10 \\
\hline Fe XI & 188.232 & 188.4 & 147.4 & 0.63 & 0.07 & G 1 & 6.01 & 6.07 & 0.71 \\
\hline $\mathrm{Fe} \mathrm{XI}$ & 188.299 & & & & & & & 6.07 & 0.25 \\
\hline Fe XII & 193.521 & 193.6 & 67.5 & 0.96 & 0.11 & G 1 & 6.12 & 6.14 & \\
\hline Fe XII & 364.467 & 364.5 & 7.8 & 1.55 & 1.09 & N 1 & 6.13 & 6.14 & \\
\hline Fe XII & 195.118 & 195.3 & 102.7 & 0.97 & 0.11 & G 1 & 6.13 & 6.14 & \\
\hline Fe XII & 192.393 & 192.4 & 14.7 & 1.43 & 0.29 & G 1 & 6.13 & 6.14 & \\
\hline Fe XIV & 274.203 & 274.2 & 40.3 & 0.86 & 0.09 & G 2 & 6.20 & 6.27 & 0.71 \\
\hline Si VII & 274.174 & & & & & & & 5.76 & 0.29 \\
\hline Fe XIII & 348.183 & 348.2 & 10.4 & 1.24 & 0.76 & N 1 & 6.22 & 6.20 & \\
\hline Fe XIII & 202.044 & 202.2 & 135.8 & 0.87 & 0.09 & G 1 & 6.23 & 6.21 & \\
\hline Fe XIV & 211.317 & 211.3 & 43.2 & 1.02 & 0.14 & G 1 & 6.29 & 6.27 & \\
\hline Fe XV & 284.160 & 284.3 & 130.3 & 1.02 & 0.10 & G 2 & 6.35 & 6.32 & \\
\hline Fe XV & 417.258 & 417.2 & 6.1 & 0.65 & 0.08 & G 3 & 6.35 & 6.32 & \\
\hline
\end{tabular}




\section{References}

Ahmad, I. A., \& Webb, D. F. 1978, Sol. Phys., 58, 323

Ahmad, I. A., \& Withbroe, G. L. 1977, Sol. Phys., 53, 397

Arnaud, M., \& Raymond, J. 1992, ApJ, 398, 394

Arnaud, M., \& Rothenflug, R. 1985, A\&AS, 60, 425

Bromage, B. J. I., et al. 1997, ESA SP-404, 241

Bromage, B. J. J., Alexander, D., Breen, A., et al. 2000, Sol. Phys., 193,181

Casalbuoni, S., del Zanna, L., Habbal, S. R., \& Velli, M. 1999, J. Geophys. Res., 104, 9947

Craig, I. J. D., \& Brown, J. C. 1986, Inverse problems in astronomy: A guide to inversion strategies for remotely sensed data (Adam Hilger, Ltd.), 159

Deforest, C. E., Hoeksema, J. T., Gurman, J. B., et al. 1997, Sol. Phys., 175,393

DeForest, C. E., Plunkett, S. P., \& Andrews, M. D. 2001, ApJ, 546, 569

Del Zanna, G. 1999, Ph.D. Thesis, Univ. of Central Lancashire, UK

Del Zanna, G. 2002, in The Radiometric Calibration of SOHO, ISSI Scientific Report, in press

Del Zanna, G., \& Bromage, B. J. I. 1999a, Space Sci. Rev., 87, 169

Del Zanna, G., \& Bromage, B. J. I. 1999b, J. Geophys. Res., 104, 9753

Del Zanna, G., \& Bromage, B. J. I. 1999c, in Proc. of the 8th SOHO Workshop: ESA-SP 446, 269

Del Zanna, G., Bromage, B. J. I., Landi, E., \& Landini, M. 2001a, A\&A, 379, 708

Del Zanna, G., Bromage, B. J. I., \& Mason, H. E. 2001b, in Solar and Galactic Composition, AIP Conf. Proc., 598, 59

Del Zanna, G., Landini, M., \& Mason, H. E. 2002, A\&A, 385, 968

Del Zanna, L., Hood, A. W., \& Longbottom, A. W. 1997, A\&A, 318, 963

Delaboudiniere, J.-P., Artzner, G. E., Brunaud, J., et al. 1995, Sol. Phys., 162, 291

Dere, K. P., Landi, E., Mason, H. E., Monsignori Fossi, B. C., \& Young, P. R. 1997, A\&AS, 125, 149

Dere, K. P., Landi, E., Young, P. R., \& Del Zanna, G. 2001, ApJS, 134, 331

Dere, K. P., Moses, J. D., Delaboudinière, J.-P., et al. 2000, Sol. Phys., 195, 13

Doschek, G. A. 1984, ApJ, 279, 446

Edgar, R. J., \& Esser, R. 2000, ApJ, 538, L167

Feldman, U. 1992, Phys. Scr, 46, 202

Fludra, A., Del Zanna, G., Alexander, D., \& Bromage, B. J. I. 1999, J. Geophys. Res., 104, 9709

Giordano, S., Antonucci, E., Noci, G., Romoli, M., \& Kohl, J. L. 2000, ApJ, 531, L79

Grevesse, N., \& Anders, E. 1991 (Solar interior and atmosphere. Tucson, AZ, University of Arizona Press), 1227
Grevesse, N., \& Sauval, A. J. 1998, Space Sci. Rev., 85, 161

Harrison, R. A. e. a. 1995, Sol. Phys., 162, 233

Hassler, D. M., Dammasch, I. E., Lemaire, P., et al. 1999, Science, 283, 810

Hassler, D. M., Wilhelm, K., Lemaire, P., \& Schuehle, U. 1997, Sol. Phys., 175, 375

Haugan, S. V. H. 1997, CDS software note, 47

Krieger, A. S., Timothy, A. F., \& Roelof, E. C. 1973, Sol. Phys., 29, 505

Laming, J. M., \& Feldman, U. 1999, ApJ, 527, 461

Lamy, P., et al. 1997, ESA SP-404, 487

Landi, E., Del Zanna, G., Breeveld, E. R., et al. 1999, A\&AS, 135, 171

Mason, H. E., \& Monsignori Fossi, B. C. M. 1994, A\&ARev, 6, 123

Mason, H. E., Young, P. R., Pike, C. D., et al. 1997, Sol. Phys., 170, 143

Mazzotta, P., Mazzitelli, G., Colafrancesco, S., \& Vittorio, N. 1998, A\&AS, 133, 403

McIntosh, S. W. 2000, ApJ, 533, 1043

Monsignori Fossi, B. C., \& Landini, M. 1991, Adv. Space Res., 11, 281

Noci, G. 1973, Sol. Phys., 28, 403

Noci, G. e. 1997, SP-404, 75

Patsourakos, S., \& Vial, J. 2000, A\&A, 359, L1

Phillips, K. J. H. 1997, Adv. Space Res., 20, 79

Raymond, J. C., Mazur, J. E., Allegrini, F., et al. 2001, in Solar and Galactic Composition, AIP Conf. Proc., 598, 49

Saito, K. 1965, PASJ, 17, 1

Schmelz, J. T., Saba, J. L. R., Ghosh, D., \& Strong, K. T. 1996, ApJ, 473, 519

Sheeley, N. R. 1996, ApJ, 469, 423

Teriaca, L., Poletto, G., Romoli, M., \& Biesecker, D., 2002, ApJ, in press

van de Hulst, H. C. 1950, Bull. Astron. Inst. Netherlands, 11, 135

Velli, M., Habbal, S. R., \& Esser, R. 1994, Space Sci. Rev., 70, 391

von Steiger, R., Schwadron, N. A., Fisk, L. A., et al. 2000, J. Geophys. Res., 105, 27217

Walker, A. B. C., Deforest, C. E., Hoover, R. B., \& Barbee, T. W. 1993, Sol. Phys., 148, 239

Wang, Y.-M. 1994, ApJ, 435, L153

Wang, Y.-M., \& Sheeley, N. R. 1995, ApJ, 446, L51

Widing, K. G., \& Feldman, U. 1989, ApJ, 344, 1046

Widing, K. G., \& Feldman, U. 1992, ApJ, 392, 715

Widing, K. G., \& Feldman, U. 1993, ApJ, 416, 392

Wilhelm, K., Marsch, E., Dwivedi, B. N., et al. 1998, ApJ, 500, 1023

Woo, R. 1996, ApJ, 464, L95

Young, P. R., Del Zanna, G., Landi, E., et al. 2002, ApJS, in press

Young, P. R., Klimchuk, J. A., \& Mason, H. E. 1999, A\&A, 350, 286

Young, P. R., \& Mason, H. E. 1998, Space Sci. Rev., 85, 315 